\title{
Satztopik und Diskurstopik in Elaboration-Kontexten
}

\author{
Michael Grabski \\ Zentrum für Allgemeine Sprachwissenschaft (ZAS) Berlin \\ grabski@zas.gwz-berlin.de
}

\begin{abstract}
The paper starts with a semantic differentiation between the notions of sentence topic and discourse topic. Sentence topic is conceived of as part of a semantic predication in the sense of Y. Kim's work. Discourse topic is defined, as in N. Asher's Segmented Discourse Representation Theory, as a discourse constituent that comprises the content of (part of) the larger discourse.

The main body of the paper serves to investigate the intricate connection between the two types of topic. For restricting the context of investigation, a specific relation between discourse constituents, Elaboration, is chosen. If Elaboration holds between two discourse constituents, one of them can be identified as the explicit discourse topic with respect to the other one. Whereas an elaborating sentence - with or without a sentence topic - is used to infer a 'dimension' for extending the discourse topic, the role of the sentence topic if it occurs is to mark an 'index' for predication along that dimension. The interaction of elaborating sentences and their topics is modelled by means of channel theoretic devices.'
\end{abstract}

\section{Kommentare als Prädikationen}

Nach Auffassung vieler Autoren ${ }^{2}$ liefern Topik und Kommentar, Subjekt und Prädikat, Fokus und Hintergrund, ebenso wie eventuelle weitere Dichotomien, Zerlegungen von Sätzen. Eine frühe Diskussion der beiden ersten Paare wird (unter einem strukturalistischen Blick) in Hocketts Buch von 1958 unternommen. Sätze werden dort prädikativen Konstruktionen zugeordnet. Für sie gilt:

The most general characteristic of predicative constructions is suggested by the terms 'topic' and 'comment' for their ICs: the speaker announces a topic and then says something about it. (Hockett 1958:201)

Diese Feststellung kann in Isolation den Eindruck vermitteln, dass Hockett die Zerlegungen 'Topik-Kommentar' und 'Subjekt-Prädikat' eigentlich nicht unterscheidet, besonders da (1) in einem ersten folgenden Beispiel durch den in immediate constituents (IC.s) zerlegten Satz in (2) illustriert wird:

1 Das Papier entstand im Rahmen der von der DFG geförderten Projekte 'Semantik-Schnittstellen: Prädikativkonstruktionen' und 'Diskurstopik' am ZAS Berlin. Für intensive Diskussion und einen Teil der Textbeispiele danke ich Werner Frey. Für hilfreiche Bemerkungen zu früheren Versionen des Papiers danke ich Ewald Lang, Claudia Maienborn und Sheila Glasbey.

2 Vgl. etwa Vallduví (1993) und die dort genannte Literatur. 
können als bestimmte Prädikationen angesehen werden. In dieser Weise ließe sich die Hockett'sche Intuition des prädikativen Charakters von TK-Gliederungen explizieren.

Durch das Topik-Suffix können im Koreanischen auch andere Konstituenten ausgezeichnet werden, etwa Adverbialphrasen ${ }^{4}$. Damit ist für diese Sprache die TK-Gliederung von der Subjekt-Prädikat-Gliederung unabhängig.

Eine andere Form der Topik-Auszeichnung liefern nach Schachter (1975) philippinische Sprachen. In ihnen zeichnet jeder Satz eine Kombination aus Topik und einem Tiefenkasus (wie actor, goal, direction, beneficient u.a.) aus. Dabei ist actor ein Tiefenkasus, der in mancher Hinsicht dem Subjekt im Englischen entspricht (Schachter a.a.O. Kap. 4). Aber auch die Topik-Auszeichnung hat 'Subjekt-Eigenschaften' (Schachter a.a.O., Kap. 2) und kann daher als 'offen' gegenüber der Subjekt-Deutung bezeichnet werden. Umgekehrt könnte im Englischen und Deutschen die Subjekt-Auszeichnung als offen gegenüber der Topik-Deutung angesehen werden.

Subjekt-NPn wären damit im Deutschen Lieferanten für Satztopiks. Sie weisen aber das Problem auf, dass sie gegenüber einer Topik-Deutung lediglich offen sind, d.h. diese allein noch nicht verlangen. Nach der Auffassung von W. Frey (vgl. Frey (2000)) können jedoch Satztopiks im Deutschen in noch anderer Weise ausgezeichnet werden, nämlich durch ihre Position im Mittelfeld des Satzes vor Satzadverbien wie vermutlich, leider, wahrscheinlich etc. Zur Illustration vgl. die Positionen des durch den Fragekontext verlangten Satztopiks Paul in (5a-c); (hier und im folgenden wird die Analyse einer Konstituente als Satztopik durch Einschluss in [ $]_{\mathrm{T}}$ verdeutlicht).

Was weisst du Neues von Paul?

(a) Nächste Woche wird [Paul] $\mathrm{T}$ vermutlich in Norwegen vortragen.

(b) ?Nächste Woche wird vermutlich Paul in Norwegen vortragen.

(c) ??Nächste Woche wird [in Norwegen $]_{T}$ vermutlich Paul vortragen.

(5b) ist schlechter als (5a), noch schlechter ist (5c), in dem der angenommene Topik-slot durch einen Referenten besetzt ist, 'nach dem nicht gefragt ist'. (5c) ist dagegen in (6b) in Ordnung als Antwort auf eine Frage wie in (6a):

(6) (a) Und wer wird nächste Woche vortragen?

(b) Nächste Woche wird [in Norwegen] T vermutlich Paul vortragen.

(6b) präsentiert ein Satztopik, das nicht Subjekt ist, nicht einmal Argumentstatus hat. Die Frey'sche Annahme kann also auch für das Deutsche einen Unterschied zwischen der TK-Gliederung und der Subjekt-Prädikat-Gliederung sichtbar machen.

Der Frage, ob es weitere Mittel der Satztopik-Auszeichnung im Deutschen gibt, wird im weiteren nicht nachgegangen. Zur Untersuchung der semantisch-pragmatischen Funktion der beiden Teile der TK-Gliederung wird diese in einen textsemantischen Zusam-

4 Ein Beispiel ist der semantische Kontrast zwischen den for-adverbials in (a) und (b), vgl. Kim (erscheint, p. 2):

(a) John-i samusil-ey han sikan-tongan issul-kkeya

John-NOM office-LOC one hour-for be-FUT

'John will be in his office for one hour.'

(b) John-i samusil-ey han sikan-tongan-un issul-kkeya

John-NOM office-LOC one hour-for-TOP be-FUT

'For one hour (from now) John will be in his office.' 
(8) (a) An diesem Tag las [Ernst]T zum Glück noch einen Teil des Aufsatzes. (b) Wie gewöhnlich fing [er]T mit dem abschließenden Kapitel an. (c) Dann machte [er]T sich einen schönen Abend. (d) Als erstes legte [er] $\mathrm{T}$ eine $\mathrm{CD}$ auf.

(b) spezifiziert weiter die in (a) erwähnte Lektüre, (c) definiert dagegen ein anderes Ereignis, das der Lektüre folgt und das seinerseits durch (d) teilweise spezifiziert wird. Intuitiv spezifiziert (d) dagegen nicht (a); auch deswegen legt (c) ein neues Diskurstopik fest. Das beibehaltene Satztopik gibt zur Erklärung dieses Wechsels nichts her.

In (7) gibt es für die Sätze (a) und (b), oder vielmehr ihre Deutungen (bezeichnet mit $\|\mathrm{a}\|$ und $\|\mathrm{b}\|$ ), nur ein implizites Diskurstopik. Symbolisieren wir dieses mit IDT und die Relation 'ist Diskurstopik von' mit einem Pfeil lässt sich der thematische Zusammenhang in (7) repräsentieren wie in

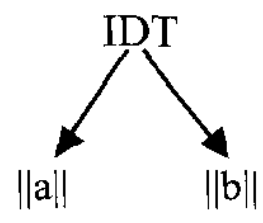

(8) erhält nach unserer Beschreibung eine Repräsentation wie in (10); danach ist $\|\mathrm{a}\|$ das Diskurstopik zu $\|\mathrm{b}\|$ und $\|\mathrm{c}\|$ das Diskurstopik zu $\|\mathrm{d}\|$. Auch (8) ist durch ein implizites Diskurstopik kohärent:

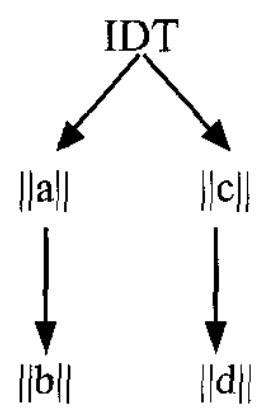

Der Intuition, dass in (8) zwischen (b) und (c) das Diskurstopik wechselt, wird in (10) dadurch entsprochen, dass $\|\mathrm{b}\|$ und $\|\mathrm{c}\|$ von verschiedenen Diskurstopiks direkt dominiert werden.

Durch eine Änderung des Textes (8) an der Stelle (d) können sich veränderte thematische Dominanzrelationen ergeben, vgl. (11); dort knüpft (d) nicht an (c) an, sondern an (a); die thematische Kohärenz dieses Textes ist dann zu repräsentieren wie in (12).

(11) (a) An diesem Tag las [Ernst $]_{T}$ zum Glück noch einen Teil des Aufsatzes. (b) Wie gewöhnlich fing [er]T mit dem abschließenden Kapitel an. (c) Dann machte [er] $\mathrm{T}$ sich einen schönen Abend. ( $\left.\mathrm{d}^{c}\right)$ Leider las [er] $\mathrm{T}$ die anderen Kapitel nicht mehr. 


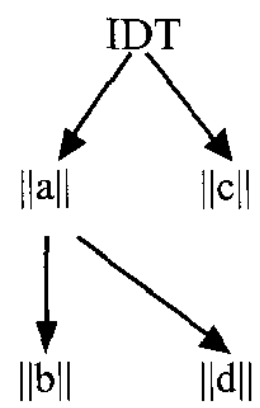

Die Pfeile in den Bäumen in (10) und (12) weisen unterschiedliche Mengen von Diskurstopiks aus, in (10) die Menge $\{\|\mathrm{a}\|,\|\mathrm{c}\|$, IDT $\}$, in (12) die Menge $\{\|\mathrm{a}\|$, IDT $\}$. Mit $\|\mathrm{a}\|$ und $\|\mathrm{c}\|$ verfügt (10) über zwei explizite Diskurstopiks, (12) nutzt dagegen $\|\mathrm{a}\|$ zweifach als explizites Diskurstopik. Dieser Unterschied lässt sich als Unterschied in der thematischen Kohärenz der Texte (8) und (11) auffassen, der durch einen Unterschied zwischen der satzinternen Information von (8d) und (11d) induziert wird. Für eine Darstellung der speziellen Relevanz der TK-Gliederung für die Textkohärenz bietet sich die Segmented Discourse Representation Theory (SDRT, vgl. Asher (1993)) an, in der systematisch der Einfluss von satzinterner Information auf die Steuerung von thematischer Kohärenz dargestellt werden kann. Im nächsten Abschnitt werden einige Konzepte der SDRT vorgestellt, die den Rahmen für die weitere Diskussion abgeben.

\section{Diskursrelationen und updating}

In SDRT werden die Deutungen der auftretenden Sätze ebenso wie auch implizite Diskurstopiks als Diskursrepräsentationen (DRS.en, vgl. Kamp (1981), Kamp/Reyle (1993)), d.h. propositionsartige Objekte ${ }^{8}$ dargestellt. Diese Objekte heißen Diskurskonstituenten.

In Übereinstimmung mit der in (10) und (12) oben verwendeten Pfeil-Notation ist 'Diskurstopik' in SDRT ein relationaler Begriff: dass eine Diskurskonstituente $\alpha$ Diskurstopik zu einer anderen Diskurskonstituente $\beta$ ist, wird damit angegeben, dass die Diskursrelation *Topic $(\alpha, \beta)$ gilt. In SDRT ist die Standard-Annahme aufgegriffen, dass die Inhalte von Texten eine hierarchische Struktur bilden (vgl. z.B. das klassische Papier von Grosz/Sidner 1986). Eine ebenfalls aufgenommene Idee ist, dass zwischen Diskurskonstituenten sog. rhetorische Relationen wie Contrast, Narration, Elaboration, Reason etc. bestehen, die einen zusätzlichen Beitrag zur 'rhetorischen Kohärenz' von Texten leisten (vgl. Mann/Thompson (1987), Polanyi (1985), Hobbs (1985), Hobbs/Agar (1985)). Rhetorische Relationen sind gleichfalls Diskursrelationen.

Nach Asher (1993) interagieren *Topic, Elaboration und Narration in spezifischer Weise: Elaboration besteht zwischen zwei Diskurskonstituenten $\alpha$ und $\beta$, wenn $\alpha$ ein explizites Diskurstopik von $\beta$ ausmacht. So gilt in (8) Elaboration $(\|\mathrm{a}||,, \mid \mathrm{b}\|)$ und Elaboration $(\|\mathrm{c}\|, \| \mathrm{d}||)$. Besteht andererseits die Relation Narration zwischen $\alpha$ und $\beta$, wird ein implizites Diskurstopik gefordert. In (8) liegt beispielsweise diese Relation zwischen $\|\mathrm{a}\|$ und $\|\mathrm{c}\|$ vor, IDT ist das hier geforderte implizite Diskurstopik.

8 Zum semantischen Status von DRSen vgl. oben Fn. 5. 
Unter dem Gesichtspunkt der sukzessiven Konstruktion (updating) einer Textrepräsentation implizieren Elaboration und Narration die Beibehaltung bzw. die Neukonstruktion von Diskurstopiks. Kennzeichnend für das updating in SDRT sind insbesondere folgende Prinzipien: ${ }^{9}$

1. Der Inhalt eines neu verarbeiteten Satzes erhält die Rolle einer Diskurskonstituente, die, anders als in der klassischen DRT, 'segmentiert' bleibt, d.h. nicht mit dem Inhalt vorhergehender Sätze verschmolzen wird.

2. Bei der Anknüpfung einer jeden neuen Diskurskonstituente $\beta$ wird die Spezifizierung einer Diskursrelation $\mathrm{R}$ gefordert.

3. Im vorhergehenden Text wird eine Diskurskonsituente $\alpha$ (attachment site) ausgewählt, an die $\beta$ durch $\mathrm{R}$ angeknüpft wird und

4. $\beta$ wird zu Zwecken der Kohärenz mit $\alpha$ ggf. um zusätzliche Information $\phi$ angereichert. Das heißt, durch Ausnutzung von Weltwissen wird eine angereicherte Konstituente $\beta^{\prime}$ inferiert.

In der skizzierten Repräsentation (10) des Textes in (8) oben ist immerhin Prinzip 1. beachtet; bei einem Ausbau zu einer vollständigen Segmentierten Diskursrepräsentationsstruktur (SDRS) wären die unspezifizierten Knoten IDT, $\|\mathrm{a}\|,\|\mathrm{b}\|$ etc. durch jeweils eine DRS zu ersetzen. Ab der Verarbeitung von (8b) wäre gemäß Prinzip 2. eine Diskursrelation zu spezifizieren (in diesem Fall Elaboration). Die Relevanz von Prinzip 3. wird durch den Unterschied zwischen der Verarbeitung von (8d) und (11d) illustriert: in (8) hat $\|\mathrm{d}\|$ als attachment site die Konstituente $\|\mathrm{c}\|$, in (11) dagegen hat $\|\mathrm{d}\|$ dafür die Konstituente $\|\mathrm{a}\|$.

Das vierte genannte Prinzip lässt sich am Text $(8 \mathrm{c}, \mathrm{d})$ illustrieren:

(8) (c) Dann machte [er]T sich einen schönen Abend. (d) Als erstes legte [er]T eine CD auf.

Dass durch $\|\mathrm{d}\|$ die Konstituente $\|\mathrm{c}\|$ elaboriert wird, ist erst gegeben, wenn $\|\mathrm{d}\|$ als Bestandteil einer Art von Ereignis gedeutet wird, wie es in (c) mit der NP ein schöner Abend gekennzeichnet wird. In (8) ist $\|\mathrm{c}\|$ attachment site für die Anknüpfung von $\|\mathrm{d}\|$ durch die Relation Elaboration. Die Relevanz des Inhaltes von \|c\| wird deutlich in (13); dort kann $\|\mathrm{b}\|$, das gleichlautend mit $\|\mathrm{d}\|$ in (8) ist, nicht mit der gleichen Diskursrelation an $\|\mathrm{a}\|$ angeknüpft werden (sondern stattdessen mit Narration). ${ }^{10}$

(13) (a) Peter kam mit seinen Einkäufen nach Haus. (b) Als erstes legte [er]T eine CD auf.

Generell berücksichtigt SDRT die Tatsache, dass zur Etablierung von Diskursrelationen zwischen geeigneten $\alpha$ und $\beta$ beim updating vier Sorten von Information ausgeschöpft werden:

9 Eine vereinfachte Version der Repräsentationserweiterung (updating) findet sich in Asher/Lascarides (1998), Abschnitt 4.

${ }^{10}$ (8d) und (13b) beziehen sich im Hinblick auf diesen Unterschied auf Information, die nicht durch presupposition trigger markiert ist. Diesem bridging-Phänomen tragen Asher/Lascarides (1998) in ihrer Definition des updating formal Rechnung. 
- Satzinterne semantische Information,

- kontextuell festgelegte Information,

- Weltwissen im weiteren Sinn und

- pragmatische Regularitäten.

Hiervon steht für uns an satzinterner Information die TK-Gliederung im Vordergrund. Andere satzinterne Information wird dagegen auf weite Strecken nur als intuitives Kriterium gehandhabt, mit dem über das Vorliegen von z.B. Elaboration entschieden wird, oder mit dem semantische Bedingungen an einen so strukturierten Text diskutiert werden. Die gleiche Art des Umgangs gilt auch für kontextelle Information und Weltwissen. Dieses Vorgehen unterscheidet sich damit von dem in SDRT, wo die verschiedenen Sorten von beteiligter Information durch Axiome in einem Logik-Modul (DICE) gegeneinander verrechnet werden. Die Intention dort ist, durch die Definition von DICE die unterschiedliche beteiligte Information semantisch vergleichbar zu machen und ihre Interaktion darzustellen.

Eine solche Darstellung soll im vorliegenden Papier noch nicht geleistet werden. Das Ziel ist, in Elaboration-Texten Abhängigkeiten zwischen der TK-Gliederung von Sätzen und einem expliziten Diskurstopik zu untersuchen und ansatzweise eine Modellierung dafür vorzuschlagen. Deren Reformulierung als Axiome im DICE-Format soll bei einer anderen Gelegenheit diskutiert werden.

Im folgenden Abschnitt werden einige grundlegende Beziehungen zwischen Satztopik, Kommentar und dem Diskurstopik diskutiert. Als ein für das weitere Vorgehen interessanter Ausgangspunkt wird in Abschnitt 5 ein Ansatz von Y. Kim zur Deutung von Satztopiks vorgestellt. Abschnitt 6 hat die Aufgabe, relevante Beobachtungen zu ElaborationTexten zusammenzustellen. In Abschnitt 7 werden ansatzweise semantische Bedingungen für die Verknüpfung einer aktualen Diskurskonstituente mit Elaboration modelliert und dabei das Auftreten von Satztopiks berïcksichtigt.

\section{Diskursrelationen und die Topik-Kommentar-Gliederung}

Die Relation Elaboration macht explizit, wie in einem Text ein Diskurstopik durch einen weiteren Satz entfaltet wird. Elaboration liefert uns damit die Möglichkeit, die Beziehung von Satztopik und -kommentar zu (expliziten) Diskurstopiks zu kontrollieren. Sei im folgenden der Satz, der das Diskurstopik repräsentiert, Kopfsatz $(\mathrm{K})$ genannt, die elaborierenden Sätze seien $E$-Sätze $(\mathrm{E} 1, \ldots)$ genannt. Es geht im folgenden um die Beziehung der TK-Gliederung von E-Sätzen zum Inhalt von (K).

Zunächst zeigt der kohärente Text in (14), dass eine Bezugnahme auf das Diskurstopik in $(\mathrm{K})$ durch die E-Sätze ggf. nur 'mittelbar' durchgeführt wird. Die Aufgabe einer solchen Bezugnahme wäre besonders beim Satztopik zu suchen, dessen Funktion, 'aboutness' zu sichern, oft betont worden ist (Reinhart (1981, 1995), Vallduví (1993, 1994), Portner/Yabushita (1998)). In (14) ist jedoch kein Satztopik in den E-Sätzen mit dem Satztopik in (K) identisch oder hat auch nur die gleiche Referenz wie eine der expliziten Konstituenten von $(\mathrm{K})$ : 
Dinner I:

(14) $(\mathrm{K})^{-}$Gestern wurde [Jörg $]_{\mathrm{T}}$ überraschenderweise wieder einmal zum Essen eingeladen.

(E1) Als erstes hat [Maria $]_{T}$ in weiser Voraussicht dem erschöpften Linguisten eine Hühnersuppe vorgesetzt.

(E2) Dann hat $[\text { sie }]_{\mathrm{T}}$ aufmerksamerweise dem Einschlafenden einen Kaffee gebracht.

(E3) Stolz trug [sie]T als nächstes dem zu Kräften Gekommenen ein Lammragout auf.

(E4) Zuletzt rundete [das Vanilleeis] $\mathrm{T}$ wie gewohnt das Mahl ab.

Die Satztopiks in (E1-E4) können als Bestandteile eines in (K) erwähnten Ereignisses (=dem Essen) inferiert werden, das selbst von dem von $(K)$ beschriebenen Ereignis (=der Einladung) verschieden ist. Dass das erwähnte Ereignis stattgefunden hat, geht in (14) allerdings erst aus den E-Sätzen hervor, genauer aus Bestandteilen der E-Sätze, die zu deren jeweiligem Satztopik komplementär sind. Die hier beobachtete Leistung der ESatz-Kommentare ist ein Anlass, ihrer Beziehung zum Diskurstopik gleichfalls Aufwerksamkeit zu widmen. Es ergeben sich drei Fragestellungen, plakativ dargestellt in (15):

1. die Beziehung zwischen (den Deutungen von) E-Satz-Topik und E-Satz-Kommentar.

2. die Beziehung (der Deutung) des E-Satz-Kommentars zum Diskurstopik

3. die Beziehung (der Deutung) eines E-Satz-Topiks zum Diskurstopik (d.h. der Deutung von $(\mathrm{K}))$

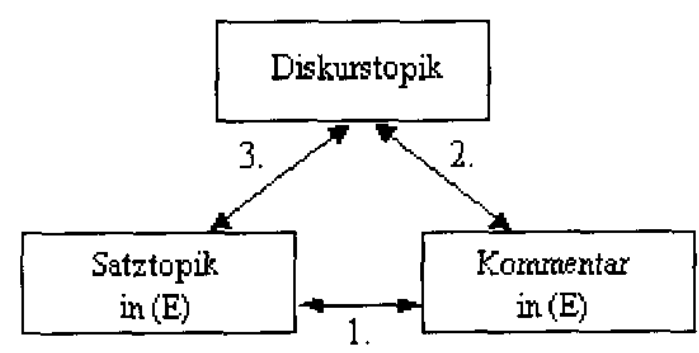

Eine Intuition zu zwei dieser Fragestellungen kann sich bei einem Blick auf die Texte in (16) und (17) einstellen. In den E-Sätzen in (17) ist gegenüber denen in (16) jeweils eine zusätzliche Konstituente aus dem Kommentar in die Satztopik-Position verschoben worden, dabei ist das Diskurstopik (die Deutung von (K)) konstant gehalten:

Fest_I_A:

(16) $(\mathrm{K})$ Gestern gab Peter ein gelungenes Fest.

(E1) Dieses Mal hatte [er $]_{\mathrm{T}}$ bemerkenswerterweise sein ganzes Geld für alkoholische Getränke ausgegeben.

(E2) Zu seiner Überraschung hatte [seine Freundin] T tatsächlich ihr tollstes Kleid angezogen.

(E3) Um ihn am Wegzug zu hindern, hatte [der Oberbürgermeister]T völlig unerwartet die Ehrenbürgerurkunde mitgebracht. 
Fest_I_B:

(17) $(\mathrm{K})^{-}$Gestern gab Peter ein gelungenes Fest.

(E1) Dieses Mal hatter [er] $\mathrm{T}$ [sein ganzes Geld] $\mathrm{T}$ bemerkenswerterweise für alkoholische Getränke ausgegeben.

(E2) Zu seiner Überraschung hatte [seine Freundin] $\mathrm{T}$ [ihr tollstes Kleid $]_{\mathrm{T}}$ tatsächlich angezogen.

(E3) Um ihn am Wegzug zu hindern, hatte [der Oberbürgermeister] $\mathrm{T}$ [die Ehrenbürgerurkunde $]_{\mathrm{T}}$ völlig unerwartet mitgebracht.

\section{zu Fragestellung 1.:}

Ein einschlägiger Kontrast zwischen (16.E1) und (17.E1) besteht etwa in folgender Weise: der Unterschied bzgl. der Kommentare geht mit dem unterschiedlichen Skopus des Satzadverbs einher. Nach (16.E1) schließt das Bemerkenswerte an Peters Verhalten auch die Verwendung der genannten maximalen Geldsumme ein, in (17.E1) nicht. Dies kann man sich verdeutlichen, wenn die Bedingungen dafür reflektiert werden, dass die Verwendung des Satzadverbs in einem der beiden Sätze nicht angebracht ist. Für (16.E1) scheint dies der Fall, wenn Peter entweder ein Verschwender ist oder gerne trinkt, für (17.E1) nur im letzten Fall. D.h. die Deutung von sein ganzes Geld spielt hier keine Rolle für die Deutung des Kommentars im 2. Satz, dagegen für die des Kommentars in (16.E1). Ein Unterschied in Bezug auf die Satztopiks in beiden Sätzen kommt durch deren Interaktion mit dem Adverbial dieses Mal heraus. (17.E1) legt die Interpretation nahe, dass bei einer anderen Gelegenheit Peter kein Geld für alkoholische Getränke, vielmehr sein ganzes Geld für etwas anderes ausgegeben hat. Diese Interpretation wird durch (16.E1) nicht nahegelegt. Nur (17.E1) vermittelt den Effekt, dass es um eine Aussage zum Schicksal von Peters ganzem Geld bei verschiedenen Gelegenheiten geht.

\section{zu Fragestellung 2,:}

Hier ist eine kontextuelle Eigenschaft der Kommentardeutung angesprochen, da das mit (K) explizit vorliegende Diskurstopik involviert ist. Ein erster Eindruck ist, dass durch die Verschiebung der Konstituenten in (17.E1-E3) eine Veränderung der 'Aspekte' einhergeht, unter denen die Deutung von (K) elaboriert wird. Die Sätze in (17.E1-E3) sind für sich betrachtet nicht unakzeptabel, charakterisieren aber das in $(\mathrm{K})$ erwähnte Fest in anderer Weise als 'gelungen' als (16.E1-E3). In (17) entsteht der Eindruck einer stärkeren Spezifik der Elaborierung, verbunden mit einer geringeren Kohärenz der E-Sätze. Die Spezifik der Elaborierung kann teilweise durch den zuvor für (17.E1) beschriebenen Effekt der Topik-Anreicherung erklärt werden: Die E-Satz-Kommentare in (17) beziehen sich auf Paare von Satztopik-Referenten; anscheinend ist für diese eine Sequenz von ESätzen schwerer kohärent zu machen als für singuläre Topik-Referenten.

\section{zu Fragestellung 3.:}

Hierzu seien die Textbeispiele (18) bis (22) betrachtet. In den E-Sätzen (18.E3) - (22.E3) sind die Satztopik-Referenten unterschiedlich verfügbar. 
Foto I A :

(18) $(\mathrm{K})$ Ich habe Anna, Klara und Paul gar nicht auf dem Foto erkannt.

(E1) Anna hatte eine schwarze Sonnenbrille auf.

(E2) Klara war durch ein Kopftuch nur halb zu sehen.

(E3) Und da [der Otto $]_{T}$ leider den Paul halb verdeckte, habe ich den Paul gar nicht erkannt.

Foto I B :

(19) $\overrightarrow{(E 3)}$ ?Und da [der Otto]T verblüffenderweise dem Paul sehr ähnelt, habe ich den Paul gar nicht erkannt.

Foto I C:

(20) $\overline{(E} \overline{3})$ ? [Otto] $\mathrm{T}$ ist ein Sohn von Paul. Otto habe ich für Paul gehalten.

Foto_I_D:

(21) $\overline{\text { (E3) }}$ Und da [der Paul $]_{\mathrm{T}}$ verblüffenderweise dem Otto sehr ähnelt, habe ich den Paul gar nicht erkannt.

Foto_I_E:

(22) $\overline{(E} 3)[$ Paul $] \mathrm{T}$ hat einen Sohn Otto. Otto habe ich für Paul gehalten.

(18.E3) und (19.E3) haben gemeinsam, dass ihre Satztopiks nicht in (K) erwähnt werden, unterscheiden sich aber in ihrer Akzeptabilität. Eine Vermutung hierzu ist, dass in (18.E3) der Satztopik-Referent Argument einer Relation VERDECKEN ist, deren Extension durch einen Bestandteil der Deutung von (K), d.h. das Foto, als definiert verstanden werden kann. In (19.E3) ist dagegen der Satztopik-Referent Argument der Relation ÄHNELN, deren Extension nicht als durch (K) definiert verstanden werden kann.

Aus dem gleichen Grund ist (20.E) schlecht: das Satztopik ist neu und Argument einer nicht durch (K) als definiert zu verstehenden Relation. Ist diese Analyse richtig, läuft sie darauf hinaus, dass in E-Sätzen solche Satztopiks nicht zugelassen sind, die nicht wenigstens durch bridging als ein Bestandteil des Diskurstopiks inferierbar sind. ${ }^{11}$

In (21.E3) und (22.E3) ist dieses Kriterium dadurch nivelliert, dass ihr Satztopik in (K) erwähnt ist, und somit nicht inferiert zu werden braucht.

Diese Beobachtungen können insgesamt zur Formulierung von Einschränkungen auf die Verfügbarkeit des Satztopik-Referenten genutzt werden. Sie geben aber noch keine Hinweise dafür her, welche Rolle die Satztopik-Referenten im Diskurstopik spielen.

"Die genannten durch $(\mathrm{K})$ definierten oder nicht definierten Relationen sind Information, die der jeweilige E-Satz-Kommentar beisteuert. 


\section{Kims Modellierung von Satztopik}

Den Unterschied zwischen den in (4a) und (4b) ausgedrückten Propositionen stellt Kim mit situationssemantischen Mitteln dar.
(4)
(a) tal-i
twungkul-ta.
Mond-NOM rund-DECL
'Der Mond ist (heute nacht) voll.'
(b) tal-un twungkul-ta.
'Der Mond ist rund.'
Mond-TOP rund-DECL

Kennzeichnend für ihre Modellierung des Kontrastes zwischen dem 'thetischen' Charakter von (4a) und dem 'kategorialen' von (4b) ist die Nutzung von vier Spezifizitäten der situationstheoretischen Ontologie. Folgende Spezifizität wird dabei jeweils folgende Nutzung erlauben:

a. Mit dem situationstheoretischen Propositionsbegriff, einer Klassifikation (vgl. Devlin (1991)), können 'unmittelbare Konstituenten' von Propositionen in variabler Weise ausgezeichnet werden: unmittelbare Konstituenten sind die Objekte, die jeweils klassifiziert werden, von Kim auch als predication base bezeichnet.

b. Als 'Austin'sche Propositionen' werden solche Propositionen bezeichnet, in denen eine Situation die unmittelbare Konstituente einer Klassifikation ist. Diese Struktur kann zu Deutung von thetischen Aussagen genutzt werden.

c. Situationen sind Objekte, die 'Information hergeben'. Wird eine Situation durch einen Typ $\tau$ klassifiziert, gilt eine konverse Relation: die Situation 'unterstützt' die von $\tau$ spezifizierte Information.

d. Der in Devlin (1991) eingeführte Situationstyp des oracle kann genutzt werden, um den Relevanz-Aspekt der TK-Gliederung zu modellieren.

Nach Kim ist (4a) eine Aussage über eine Situation, (4b) dagegen eine solche über ein Objekt (den Mond). Nach Spezifizität a. und b. kann in (4a) die mit der Satzäußerung intendierte Situation s als unmittelbare Konstituente der ausgedrückten Proposition aufgefasst werden; das heißt, dass es eine Eigenschaft von s ist, dass der Mond voll ist. Diese Deutung lässt sich repräsentieren wie in ((23); dort ist ':' zu lesen als 'ist vom Typ':

$$
\mathrm{s}: \mathrm{RUND}(\mathrm{m})
$$

Eine solche Proposition entspricht nach Kim einer 'thetischen' Deutung, da sie die Klassifikation einer Situation s behauptet. In diesem Fall ist s die 'unmittelbare Konstituente' (oder predication base) der Proposition. In (4b) ist dagegen das Objekt Mond als unmittelbare Konstituente der Deutung anzusetzen. Eine Repräsentation für (4b) hat die Struktur (24); dabei ist der Typ $\tau$, nach dem m klassifiziert wird, weiter zu spezifizieren:

$$
\mathrm{m}: \tau
$$

Kims Vorschlag dazu ist, $\tau$ durch Abstraktion aus einer Proposition zu konstruieren, die die Relevanz der Klassifikation für das betr. Objekt (den Mond) sichert. In Devlin (1991) 
wird ein Typ von Situationen definiert, der für ein gegebenes Objekt die vollständige faktische - jedoch nicht notwendigerweise bekannte - Information zu diesem Objekt enthält; für ein Objekt $\mathrm{x}$ wird eine solche Situation mit oracle $_{x}$ bezeichnet. Um anzugeben, dass es Teil der vollständigen faktischen Information zum Mond ist, dass er rund ist, läßt sich eine Proposition wie in (25a) angeben. Durch $\lambda$-Abstraktion lässt sich hieraus für den vorliegenden Zweck ein Typ wie (25b) definieren, intuitiv der Typ aller Dinge, für die gilt, dass ihr Rundsein Bestandteil der faktischen Information über sie ist. Eine semantische Entsprechung zu (4b) ist dann (25c), in dem behauptet wird, dass $m$ von diesem Typ ist.
(a) oracle $_{x}: \mathrm{RUND}(\mathrm{x})$
(b) $\lambda \mathrm{x}\left[\right.$ oracle $\left._{x}: \mathrm{RUND}(\mathrm{x})\right]$
(c) $\mathrm{m}: \lambda \mathrm{x}\left[\right.$ oracle $\left._{x}: \mathrm{RUND}(\mathrm{x})\right]$

Der Typ $\lambda x\left[\right.$ oracle $\left._{x}: \operatorname{RUND}(\mathrm{x})\right]$ ist ein wesentlicher Teil des Kommentars von (4b); im Rahmen der TK-Gliederung von (4b) spezifiziert der Kommentar 'neue Information'. Die Klassifikation der oracle $_{x}$-Situation durch RUND(x) liefert nun Kim eine Art Einschlägigkeit der neuen Information zu dem Objekt, das den Parameter x belegt. Damit dass in (25c) dem Objekt $m$ die Eigenschaft zugeschrieben wird, dass 'sein' oracle die Information unterstützt, dass RUND(x) gilt, ist RUND(x) als Teil der faktischen Gesamtinformation zu $\mathrm{m}$ ausgezeichnet.

Interessant ist an dieser Lösung, dass es gerade die Deutung des Kommentars ist, die zum Satztopik etwas Relevantes beiträgt und damit 'für das Satztopik aboutness leistet'.

Es stellt sich allerdings die Frage, ob die für diese Konstruktion benötigte ausgezeichnete Situation allgemein mithilfe des oracle-Konzepts festgelegt werden sollte. Ein Kontext, in dem eine Äußerung von (4b) informativ ist, ist etwa eine Situation, in dem der Mond gerade nicht voll ist, d.h. nicht rund aussieht. Dann ist oracle $_{m}$ die ausgezeichnete Situation, die die eigentliche Gestalt des Objekts spezifiziert. Aber Relevanz des Kommentars muß nicht in einer Klassifikation durch das Wahre-aber-nicht-Evidente bestehen. Dass der Kommentar in dieser Hinsicht relevant ist, scheint eher kontextspezifisch bedingt. Akzeptiert man, dass Satzadverbien einen Teil des Kommentars ausmachen und dass sie die im Kommentar enthaltene ausgezeichnete Situation mitbestimmen, ist ihre semantische Vielfalt ein Argument dafür, dass eine Relevanz des Kommentars in variablerer Weise möglich ist.

Mit dem Satzadverb eigentlich kann etwa die in (4b) ausgedrückte Relevanz im Deutschen explizit gemacht werden, vgl. (26):

Der Mond ist eigentlich rund.

Zählt man nun mit Lang (1979) auch einen Operator wie wahrscheinlich zu den Satzadverbien, und schreibt man ihm mit Frey eine Satztopik-Auszeichnung zu, dann ist eine nicht notwendigerweise faktive Situation in Betracht zu ziehen.

Bei der Gelegenheit hat [Hans]T wahrscheinlich Maria geküsst.

Bei unmarkierter Intonation ist Hans hier Satztopik. Die dann durch wahrscheinlich ausgesonderte Situation (in der Hans Maria küsst) ist nicht notwendigerweise faktiv; damit 
kann sie nicht ein Teil von oracle Hans sein, denn für letzteres wird von Devlin verlangt, dass es realisiert (d.h. Teil der aktualen Welt) ist.

Es scheint daher sinnvoll, Kommentar-Relevanz in einer allgemeineren Weise zu modellieren. Diese sollte mit einem Beitrag von Satzadverbien verträglich sein, der nicht auf die oracle-Konstruktion eingeschränkt ist, sondern kontextueller Abhängigkeit Rechnung trägt. Unten wird diese dadurch dargestellt, dass als Relevanz stiftende Situation diejenige Situation fungiert, die das Diskurstopik unterstützt.

\section{Elaboration-Kontexte}

Zur Diskussion der Beziehung zwischen TK-Gliederung und Diskurstopik kehren wir zur Betrachtung von Texten zurück, die durch Elaboration strukturiert sind. Beispiele für solche Texte zeigten in Abschnitt 4., dass es Bezugnahmen von Satztopiks in E-Sätzen auf das Diskurstopik gibt, die aber 'mittelbar' sein können:

- Satztopiks in (E) wurden nicht notwendigerweise von Konstituenten von (K) geerbt, erst recht nicht notwendigerweise vom dortigen Satztopik (vgl. (14));

- die Rolle von Satztopiks in E-Satz-Sequenzen ist intuitiv an eine Folge von Aspekten geknüpft, in Bezug auf die das Diskurstopik elaboriert wird $(16,17)$;

- Satztopiks im E-Satz müssen als Bestandteil der Diskurstopik-Situation inferierbar sein $(18-22)$.

Damit verfügen wir zunächst nur über eine unzusammenhängende Charakterisierung der Beziehung zwischen den beiden Topik-Sorten. In diesem Abschnitt sollen weitere Bedingungen für die Kohärenz von Elaboration-Texten formuliert werden. Ziel ist dabei, die Klasse von Verarbeitungskontexten einzuschränken, die die Wahl der Diskursrelation Elaboration legimieren und in denen die spezifische Funktion von Satztopik angegeben werden kann.

\subsection{Topiklose E-Sätze}

Zunächst ist festzustellen, dass die E-Sätze nicht notwendigerweise Satztopiks enthalten. Dies ist der Fall in (28):

Wetter_ I:

(28) (K) Gestern war ein unbeschreibliches Wetter.

(E1) Zuerst war es sehr heiß.

(E2) Dann stürmte es.

(E3) Schließlich fing es an zu schneien.

Satztopiks können nicht zur Erklärung der Intuition herangezogen werden, dass in (28) (E1) bis (E3) etwas zur Aussage von $(\mathrm{K})$ beisteuern, diese sogar 'elaborieren'. Immerhin finden wir in (E1) bis (E3) 'kontrastierende Aspekte' auf die in (K) gemachte Aussage. Die Existenz dieser Aspekte wollen wir im folgenden im Begriff einer Dimension zusammenfassen, entlang der die Aussag in (K) elaboriert wird. Im Beispiel (28) erhält diese Dimension eine Struktur durch die von den Adverbien zuerst, dann und schließlich 
angesprochene Zeitachse. ${ }^{12}$ Es wird sich zeigen, dass mit dieser (für Elaboration-Texte grundlegenden) Struktur Satztopiks interagieren, wenn sie auftreten.

\subsection{Dimensionen, Indizes und Positionen}

Zur Diskussion dieses Zusammenhangs sei wieder von Texten ausgegangen, in denen (K) und E-Sätze über Satztopiks verfügen. Vgl. als erstes:

Schulwahl_I_A:

(29) (K) Nach dem 6. Schuljahr suchen die Freunde von Meryem leider verschiedene Schulen auf.

(E1) So wird Hamdi glücklicherweise aufs Gymnasium gehen.

(E2) Dagegen geht Arkan vermutlich auf die Realschule.

(E3) Trotz ihrer Begabung wird Songül leider die Hauptschule besuchen müssen.

In Schulwahl_I_A enthält (K) zwei Konstituenten mit pluralischen Referenten (die Freunde von Meryem, verschiedene Schulen), auf die sich die E-Sätze beziehen. Auf den pluralischen Referenten der zweiten Konstituente (verschiedene Schulen) wird in den ESätzen jeweils durch Erwähnung eines seiner Elemente Bezug genommen. Diese Bezugnahmen sind insofern deskriptiv vergleichbar, als auf die jeweilige Schule durch ihren Schultyp referiert wird. 'Schultypen' lässt sich hier als die Dimension auffassen, entlang der eine in $(\mathrm{K})$ gemachte Aussage, nämlich dass verschiedene Schulen besucht werden, elaboriert wird. Die einzelnen Schultypen kann man mit Positionen auf der gegebenen Dimension identifizieren, Bündel von Eigenschaften, die sie vergleichbar und paarweise disjunkt machen. Die Funktion der ersten Konstituente (die Freunde von Meryem) kann dagegen als Bereitstellung einer Menge von Indizes gesehen werden. Mit dem Bezug auf einen Index durch das jeweilige Satztopik wird in den E-Sätzen in (29) ein jeweils verschiedener Aspekt auf das elaborierte Objekt artikuliert. In diesem Text kann die zugrundeliegende Dimension als Folge von Paaren aus einem Index und der zugehörigen Position aufgefasst werden.

Mit der Deutung von die Freunde von Meryem als 'indexstiftende' bzw. von verschiedene Schulen als 'positionsstiftende' Konstituente von (29.K) wird eine Asymmetrie behauptet. Kann in (29) nicht auch verschiedene Schulen als die indexstiftende Konstituente analysiert werden? Dann würden durch die Eigennamen Hamdi etc. nunmehr Positionen auf einer von den E-Sätzen etablierten Dimension angegeben. Eine solche Lesart der ESätze von (29) sollte damit verträglich sein, dass entsprechend die Schultypen als gegebene Information, die jeweíligen Schüler als neue Information präsentiert. Dies läßt sich dadurch erreichen, dass in $(\mathrm{K})$ auf die Schultypen aus einer Satztopik-Position Bezug genommen wird, konstruierbar durch eine Passivierung, vgl.

Nach dem 6. Schuljahr werden verschiedene Schulen wahrscheinlich von den Freunden von Meryem aufgesucht.

Die Satztopik-Position ist hier durch das Satzadverb wahrscheinlich markiert. Für die intendierte Verwendung als K-Satz passen wir die auf die Schultypen referierende Kon-

12 Das gleiche gilt für das Beispiel in (14), Dinner_I. Die 'Fest-Beispiele' (16, 17) verlangen die Konstruktion einer abtrakteren Dimension, etwa 'betriebener Aufwand', vgl. unten Abschnitt ... Die Dimension in den 'Foto-Beispielen' ist auf den beteiligten Personen definiert. 
stituente noch in einer weiteren Hinsicht an: wir machen sie definit wie in (31.K), da eine indefinite Version wie in (30) nicht die distributive Lesart haben kann.

Schulwahl_I_B:

(31) (K) Nach dem 6. Schuljahr werden die verschiedenen Schulen wahrscheinlich von den Freunden von Meryem aufgesucht.

(E1) So wird Hamdi glücklicherweise aufs Gymnasium gehen.

(E2) Dagegen geht Arkan vermutlich auf die Realschule.

(E3) Trotz ihrer Begabung wird Songül leider die Hauptschule besuchen müssen.

Es zeigt sich, dass die resultierende Abfolge von (31.K) und (31.E1-E3) jedoch nicht gut ist. Intuitiv gibt es einen 'Sprung' zwischen (31.K) und (31.E1), der sich als Wechsel zwischen den intendierten Rollen von Schultypen (in (K): Indizes) und Schülern (in (K): Positionen) erklären lässt. In dieser Hinsicht in Ordnung scheint dagegen der Text in (32), in dem in den Satztopik-Positionen der E-Sätze sich solche Ausdrücke befinden, die auf die intendierten Indizes (die Schultypen) Bezug nehmen.

Schulwahl_I_C:

(32) (K') Nach dem 6. Schuljahr werden die verschiedenen Schulen wahrscheinlich von den Freunden von Meryem aufgesucht.

(E1) So wird aufs Gymnasium glücklicherweise Hamdi gehen.

(E2) Dagegen geht auf die Realschule vermutlich Arkan.

(E3) Trotz ihrer Begabung wird die Hauptschule leider Songül besuchen müssen.

Die Diskussion der Beispiele (29), (31) und (32) legt nahe, in E-Sätzen die Deutung der Satztopiks als Artikulation von Indizes und die Deutung von Kommentaren als Differenzierung von Positionen aufzufassen. Diese Beispiele sind nun in einer Reihe von Hinsichten idealisiert, die unten diskutiert werden sollen. Wie sie stehen, lässt sich an ihnen aber eine generllere Entsprechung zwischen K- und E-Sätzen behaupten. die darin besteht, dass durch die TK-Gliederung der E-Sätze auf eine Prädikation in (K) Bezug genommen wird. Was in (29.K) für Mengen von Schülern behauptet wird, wird in den nachfolgenden E-Sätzen für einzelne Schüler spezifiziert. Deutet man (wie Kim) die TK-Gliederung der E-Sätze selbst als eine Prädikation, läuft das darauf hinaus, dass in (29) und (32) das Bestehen von Elaboration zwischen $(\mathrm{K})$ und den E-Sätzen als eine Relation zwischen Prädikationen erkärt werden kann. Dass (31) weniger kohärent ist, kann auf das NichtVorliegen dieser Relation zurückgeführt werden.

Diese Annahme lässt sich auch durch Beobachtungen zu Texten stützen, in denen explizite Indizes fehlen. In bestimmten Fällen können spezifische E-Sätze spezifische Prädikationen in $(\mathrm{K})$ verlangen, vgl. die beiden folgenden Texte:

Wetter II:

(33) (K) Es regnete.

(E1) Viele kleine Tropfen wirbelten herab.

(E2) Auch die wetterabgewandten Seiten der Baumstämme wurden nass.

Wetter_III:

(34) (K) Es regnete anders als sonst.

(E1) Viele kleine Tropfen wirbelten herab.

(E2) Auch die wetterabgewandten Seiten der Baumstämme wurden nass. 
In Wetter_II liefern die Sätze (E1) und (E2) Diskurskonstituenten, für die die Diskursrelation Cause besteht. Zu der hieraus zu konstruierenden komplexen Konstituente hat die Deutung von (K) eher die Relation Background. In Wetter_I I I wird dagegen in (K) die VP durch ein manner-Adverb modifiziert. In diesem Fall scheint eine Anknüpfung mit Elaboration möglich.

Die Idee einer Relation zwischen Prädikationen werden wir unten zur Modellierung der Diskursrelation Elaboration und ihrer Interaktion mit Satztopiks verwenden. Ein Beispiel wie (28) zeigt jedoch, dass in E-Sätzen nicht notwendigerweise Indizes in Form von Satztopiks vorkommen müssen. In den folgenden Abschnitten werden Typen von Elaborationstexten vorgestellt, die von den Beispielen (29) und (32) in verschiedener Hinsicht abweichen.

\subsection{Inferierte Positionen}

Das oben bereits angeführte Beispiel Fest I A weist eine Dimension auf, für die es in (K) keine index- oder positionsstiftende Konstituente gibt. Was die Positionen sind, ist aus der Art und Weise zu inferieren, in der die Kommentare der E-Sätze vergleichbar sind; Rahmen für die Elaboration ist nicht die gesamte Deutung von (K), sondern der Referent der NP ein gelungenes Fest.

Fest_I_A:

(16) $(\bar{K})$ Gestern gab Peter ein gelungenes Fest.

(E1) Dieses Mal hatte [er] $\mathrm{T}$ bemerkenswerterweise sein ganzes Geld für alkoholische Getränke ausgegeben.

(E2) $\mathrm{Zu}$ seiner Überraschung hatte [seine Freundin] T tatsächlich ihr tollstes Kleid angezogen.

(E3) Um ihn am Wegzug zu hindern, hatte [der Oberbürgermeister]T völlig unerwartet die Ehrenbürgerurkunde mitgebracht.

Als Dimension ist etwa inferierbar "Art von Aufwand für das Fest". Als Positionen können hier idealisierte Konzepte angesetzt werden, etwa: 'Getränke', 'Kleidung' und 'Sozial relevante Ereignisse', für die eine Kontrastbeziehung besteht.

$\mathrm{Zu}$ beobachten ist, dass die Polarität der Prädikation in (16.K), nämlich dass es sich um ein 'gelungenes' Fest handelt, geändert werden kann, bei Annahme einer symmetrischen Dimension (und bei entsprechender Modifikation der E-Sätze):

Fest II:

(35) (K) Gestern gab Peter ein misslungenes Fest.

(E1) Dieses Mal hatte er geizigerweise nur wenig Geld für die Getränke ausgegeben.

(E2) $\mathrm{Zu}$ seinem Verdruss hatte seine Freundin leider ihr unvorteilhaftestes Kleid angezogen.

(E3) Ärgerlicherweise brachte eine Polizeistreife völlig unerwartet Knöllchen an den Autos mehrerer Gäste an.

Auch zu inferierende Dimensionen sind nicht auf die Benutzung von Indizes in Satztopik-Position angewiesen, vgl.: 
Fest III:

(36) (K) Gestern gab Peter ein gelungenes Fest.

(E1) Glücklicherweise hatte er genug Getränke besorgt.

(E2) Tatsächlich hatte seine Freundin ihr tollstes Kleid angezogen.

(E3) Völlig unerwartet brachte der Oberbürgermeister die Ehrenbürgerurkunde mit.

\subsection{Nicht-konstrastierende Positionen}

In einem Elaborationstext wie (37) besteht Kontrast zwischen den E-Sätzen nur hinsichtlich ihrer Subjekte, für die ein Satztopik-Status angenommen werden kann. Die E-SatzKommentare spezifizieren keine kontrastierenden Positionen.

Gestirne:

(37) (K) Die Gestirne habe alle die gleiche Gestalt.

(E1) Der Mond ist rund.

(E2) Die Erde ist ebenfalls rund.

(E3) Auch die Sonne ist rund.

\subsection{Keine disjunkten Teilobjekte}

In (38) macht (K) kein Objekt explizit, das für die E-Sätze einzeln spezifizierbare TeilObjekte liefert. Kontrast wird dort nur durch die Prädikate erzeugt.

Wetter_1:

(38) $(\mathrm{K})^{-}$Es war gutes Wetter.

(E1) Es war nicht zu kalt.

(E2) Und es war nicht zu trocken.

\subsection{Gemischter Kontrast}

In (39) spezifiziert nur einer von zwei E-Sätzen, (E2), ein explizites Objekt, über das prädiziert wird. (E1) nennt dagegen ein 0-stelliges Prädikat:

LL-Wetter:

(39) (K) Es war gutes Langlaufwetter.

(E1) Es regnete nicht.

(E2) Die Loipe war nicht vereist.

Vergleichbarkeit der Dimensionspositionen kann hier auf zwei Wegen erfolgen: entweder werden Wettereigenschaften verglichen; dann ist aus (E2) zu inferieren, dass es nicht zu kalt war. Oder eine Vergleichbarkeit hinsichtlich Umgebungsbestandteilen wird konstruiert; dann werden Luft und Boden verglichen.

\subsection{Intentionalität von Dimensionen}

Ein weiterer Aspekt der Dimensionskonstruktion ist, dass ein gegebener K-Satz mehrere Dimensionen zulässt. In Schulwahl_ II kann die Dimension beschrieben werden als "Ort der Schule (im Hinblick auf die Wohnung)". Dass es weitere Möglichkeiten gibt, 
kann durch ein Beispiel wie Schulwahl_ I I I belegt werden, in dem es um architektonische Kontraste geht.

Schulwahl II:

(40) (K) Nach dem 6.Schuljahr gehen die Freunde von Meryem auf verschiedene Schulen:

(E1) So wird Hamdi auf ein Gymnasium in seinem Stadtbezirk gehen.

(E2) Arkan geht vermutlich auf das Türkische Gymnasium im angrenzenden Bezirk.

(E3) Trotz seiner Entfernung wird Songül das Naturwissenschaftliche Gymnasium besuchen.

Schulwahl III:

(41) (K) Nach dem 6.Schuljahr gehen die Freunde von Meryem bedauerlicherweise auf verschiedene Schulen:

(E1) So wird Hamdi glücklicherweise auf eine Schule gehen, die kürzlich im Rahmen eines Architekturwettbewerbs gebaut wurde.

(E2) Dagegen geht Arkan vermutlich auf ein Gymnasium, das in einem Nachkriegsneubau untergebracht ist..

(E3) Trotz ihres Asthmas wird Songül leider in einem klimatisch bedenklichen Altbau bleiben müssen.

\subsection{Andere Dimensionsauszeichnungen}

Mit dem Dimensionsbegriff kann auch eine andere Form der Auszeichnung von topikaler Information in Zusammenhang gebracht werden. Diese besteht in der Kennzeichnung von Indizes durch als-Phrasen, vgl.:

Dinner II:

(42) $(\mathrm{K})$ Gestern wurde Jörg überraschenderweise zum Essen eingeladen.

(E1) Als Vorspeise hat Maria dem erschöpften Linguisten eine Hühnersuppe vorgesetzt.

(E2) Als Hauptgang trug sie dem zu Kräften Gekommenen ein Lammragout auf.

\subsection{Nur ein E-Satz}

Bei der Verarbeitung von Elaboration-Texten tritt regelmäßig die Situation auf, dass für einen Satz dessen Status als erster E-Satz zu bestimmen ist. D.h. es ist im gegebenen Fall für einen Satz wie (E1) in (43) festzustellen, dass seine Deutung zu der von (43.K) in der Relation Elaboration steht. Kontraststiftende weitere E-Sätze sind, selbst wenn vorhanden, dann noch nicht verarbeitet und sind deshalb semantisch nicht nutzbar.

Wetter_2:

(43) (K) Es regnete.

(E1) Es fielen ungewöhnlich dicke Tropfen. 


\section{Modellierung}

Begriffe wie 'Dimension' und 'Index' sollen nun dadurch präzisiert werden, dass die Bedingungen für das Vorliegen von Elaboration in Bezug auf einige beispielübergreifende Phänomene modelliert werden.

\subsection{Dimensionen in Feldern}

Die Variation der Dimensionswahl wie in den 'Schulwahl'-Beispielen ist im konkreten Fall sicher durch die Notwendigkeit bedingt, eine Klassifikation nach einem kontextuell bedingten Gesichtspunkt vorzunehmen. In der Semantik ist an einer anderen Stelle die Variation von Klassifikationen diskutiert worden, und zwar in Ansätzen zur Wortfeldanalyse, vgl. Lutzeier (1981). Es geht dort um die Feststellung semantischer Kontraste zwischen Lexemen, die in einem gegebenen verbalen Kontext (einem semantisch eingeschränkten Paradigma) auftreten können; ein Beispiel folgt weiter unten. Die Festlegung solcher Kontraste kann - außer der Berücksichtigung von sog. semantischen Relationen zwischen Lexemen (Hyponymie, Hyperonymie, Antonymie etc.) - die semantische Klassifikation von Lexemen nach deskriptiven Kriterien erfordern.

Die Klassifikationen werden entlang von Dimensionen vorgenommen. Eine Dimension definiert die Zerlegung einer Menge von Objekten (im Falle der Wortfelder von Lexemen). Zur Individuierung der Elemente einer gegebenen Menge können mehrere Dimensionen erforderlich sein, und ihre Verwendung kann nach operativen Gesichtspunkten erfolgen (Lutzeier 1981).

Eine schematische Darstellung dieses Vorgehens ist in (44) skizziert. Bei einer Menge von Objekten $\mathrm{G}=\left\{\mathrm{o}_{1}, \mathrm{o}_{2}, \ldots, 0_{7}\right\}$ ist eine mögliche Lösung der Aufgabe, jedes Element von $\mathrm{G}$ von jedem anderen deskriptiv zu unterscheiden, die Verwendung von 3 Dimensionen D1, D2 und D3. Jede von ihnen zerlegt $G$ in sog. Zerlegungsmengen. In (44) zerlegt z.B. die Dimension D1 die Menge $G$ in Zerlegungsmengen mit den Namen $z_{11}, z_{12}$ und $z_{13}$ :

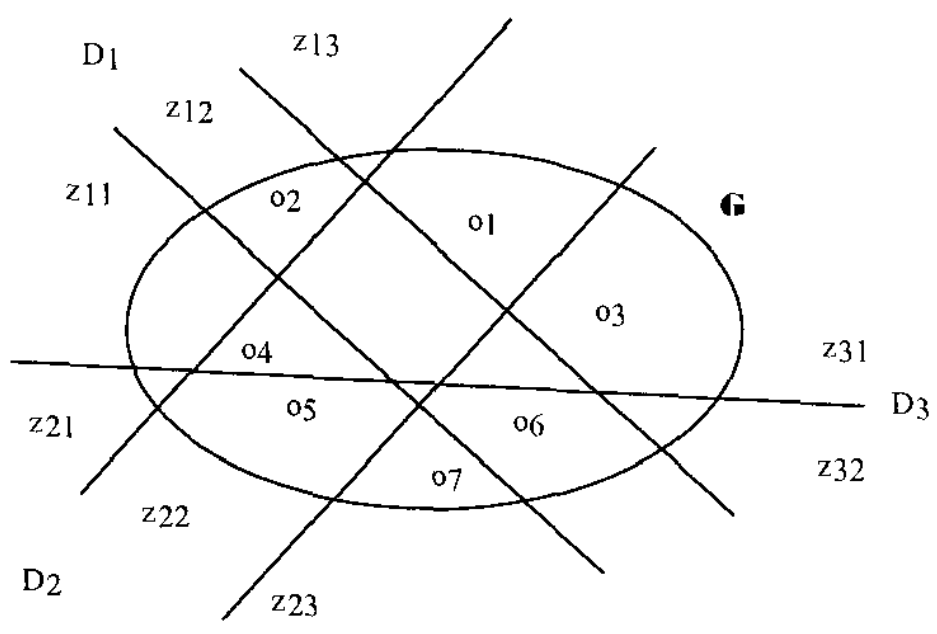

Der Sinn von Zerlegungen von Wortfeldern nach Dimensionen lässt sich an zwei konkreten Beispielen belegen: 
1. Verwandtschaftsbezeichnungen: In Lutzeier (1981) findet sich eine Analyse der Verwandtschaftsbezeichnungen im Deutschen. Aus einem angenommenen 'verbalen Kontext' $Y$ kann ich zu meiner Verwandtschaft zählen, der Festlegung einer syntaktischen Kategorie Substantiv und eines 'semantischen Aspektes' Generelle Verwandtschaftsbezeichnung bezüglich Ego ergibt sich eine Menge von Lexemen $\mathrm{G}=\{$ ururgroßvater, ururgroßmutter, ..., urenkelin $\}$ mit mehr als vierzig Elementen (vgl. p. 160). Diese werden mit nur drei Dimensionen individuiert, die allerdings z.T. intern reich strukturiert sind. Dies gilt besonders für die erste verwendete Dimension, "Abstand in Generationen zu Ego", die u.a. die folgenden Zerlegungsmengen mit ihren Namen spezifiziert:

$$
\begin{aligned}
& \ddot{Z}_{1-2}=\{\text { enkel, enkelin }\} \text { mit } N_{1-2}=\underline{\text { Zwei Generationen nach Ego }} \\
& \dddot{\mathrm{Z}}_{10}=\{\text { bruder, schwester, geschwister, ..., stiefschwester }\} \text { mit } \mathrm{N}_{10}=\underline{\text { Gleiche }} \\
& \mathrm{Z}_{11}=\{\text { vater, mutter, ..., schwiegereltern }\} \text { mit } \mathrm{N}_{11}=\text { Eine Generationen vor } \\
& \text { Ego }
\end{aligned}
$$

Die beiden anderen Dimensionen klassifizieren die Lexeme in $G$ nach 'Geschlechtszugehörigkeit' und nach 'Familienzugehörigkeit durch Heirat'. Eine Komplexität ist bei der letzten Dimension, dass Zugehörigkeit durch Heirat generationsmäßig differenziert werden muss, vgl. den Kontrast zwischen schwiegersohn und stiefbruder (Lutzeier 1981:162).

Bemerkenswert an diesem Wortfeld erscheint zum einen die Effizienz der Dimensionen. Sie reichen beim vorliegenden Beispiel für die Individuierung der Lexeme aus, semantische Relationen spielen nach Lutzeiers Analyse keine Rolle. Die benutzten Kriterien dürften bei diesem Wortfeld lexikalisiert sein.

Ein zweiter Punkt ist, dass Dimensionen relativ komplex definiert sein können, wie für die 3. Dimension angedeutet. Mit der Bereitstellung von 'Namen für die Zerlegungsmengen', vgl. $\mathrm{N}_{1-2}$ etc. in (45) wird in dem Ansatz ein möglicher sprachlicher Zugriff auf Bestandteile von Klassifikationen vorgestelit. Dieser Gedanke soll unten (Abschnitt 7.6) genutzt werden.

2. Bezeichnungen für Speisen. Allgemein liefern Bezeichnungen für Artefakte Wortfelder, die sinnvoll durch Dimensionen individuiert werden. ${ }^{13}$ An Bezeichnungen für Speisen läßt sich zeigen, dass die Einschlägigkeit von Klassifikationen kontextuell stark variieren kann. Die Klassifikation von Speisen nach bestimmten Ingredienzien, Zubereitungsweisen, Anbindungen an institutionalisierte Gelegenheiten (Feste) etc. kann je nach Gelegenheit relevant oder irrelevant sein. Eine spezielle Klasse von Kontexten ist z.B. mit der Bezeichnung 'Diät' verbunden; in einem solchen Kontext wird ein angenommener physischer Zustand eines Individuums mit einer Klassifikation von Speisen nach ihrer Zuträglichkeit korreliert. Weitere Faktoren wie Konstitution des Individuums oder Ressourcen an Zutaten können zu einer (diätbezogenen) temporären Klassifikation, etwa in einer Liste von vorgeschlagenen Speisen, führen. Maßgeblich ist dann eine Dimension mit Zerlegungsmengen, die mit Bezeichnungen wie zuträglich, noch erlaubt, schädlich, ... gekennzeichnet werden können.

${ }^{13} \mathrm{Vgl}$. Lutzeiers Beispiel 'Bezeichnungen für Turngeräte', a.a.O. Kap. 5.1.1. 
Dimensionen, wie sie bei Lutzeier modelliert sind, nämlich als Indexmengen für Zerlegungen von Mengen, scheinen geeignet, zur Explikation des bisher lose verwendeten Dimensionsbegriff beizutragen. Attraktiv an dieser Definition ist die Tatsache, dass die durch jeweilige Zerlegung entstandenen Mengen

- nach kontrastierender deskriptiver Information unterschieden werden,

- mit sprachlichen Mitteln gekennzeichnet werden können und

- einen kontextabhängigen ('temporären') Status haben.

Aus dem in der Wortfeld-Definition ${ }^{14}$ angelegten sprachlichen Zugriff auf die Zerlegungsmengen können wir eine Deutung von rahmensetzenden Adjunkten wie im Text Dinner_I I ableiten; die Adjunkte als Vorspeise und als Hauptgang können als Kennzeichnung von Positionen auf der Dimension interpretiert werden, nach der die E-Sätze den K-Satz elaborieren.

Dinner II:

(42) (K) Gestern wurde Jörg überraschenderweise zum Essen eingeladen.

(E1) Als Vorspeise hat Maria dem erschöpften Linguisten eine Hühnersuppe vorgesetzt.

(E2) Als Hauptgang trug sie dem zu Kräften Gekommenen ein Lammragout auf.

Andere topikale Information wird durch die Satztopiks selbst gegeben. Durch sie wird nicht eine Position im eigentlichen Sinn gekennzeichnet. Die Auszeichnung kontrastierender Positionen einer Dimension wurde oben als Leistung der Kommentar-Deutungen beschrieben. Satztopiks lieferten dafür 'Indizes'. Beispiele wie (28) (Wetter_I) zeigten allerdings, dass Dimensionen, die für die Kohärenz von E-Satz-Sequenzen sorgen, nicht auf das Vorkommen von Satztopiks angewiesen sind. Es reicht ggf. KommentarInformation, um die temporäre Klassifikation zu etablieren.

\subsection{Channels}

Die Modellierungsidee, der wir nun folgen wollen, berücksichtigt den inferierten Status der jeweiligen Dimension. Sie berücksichtigt ferner die Tatsache, dass die durch eine Dimension strukturierte Klassifikation temporär ist. (Dies ist gerade deswegen der Fall, weil sie auf Propositionen basiert, d.h. auf semantischen Objekten, die durch sprachliche Komposition beliebig komplex gemacht werden können.) Die temporären Klassifikationen beruhen auf (durch E-Sätze gemachte) Aussagen zu einem Bestandteil eines spezifischen Diskurstopiks. Sie definieren einen spezifischen Informationsfluss zwischen den Inhalten der E-Sätze und einem relevanten Bestandteil des Diskurstopiks.

Die zentrale Aufgabe, die bei der Verarbeitung eines E-Satzes zu leisten ist, kann nun so interpretiert werden: die von einem 'putativen E-Satz' ausgedrückte Proposition ist daraufhin zu prüfen, ob sie ein Teil einer temporären Klassifikation (Dimension) ist, der sich auf einen relevanten Bestandteil von $\|\mathbf{K}\|$ bezieht.

Zur Darstellung dieser Aufgabe lässt sich die von Barwise und Seligman entwickelte Channel Theory verwenden. Sie berücksichtigt zwei Aspekte, unter denen temporäre Klassifikationen partial sind: sie gelten normalerweise für einen eingeschränkten Bereich von Objekten (Tokens) und sie klassifizieren diese Objekte nach einer eingeschränkten

\footnotetext{
${ }^{14}$ Diese wird hier nicht präzisiert; vgl. dazu Lutzeier (1981), Kap. 4.7.
} 
Menge von Typen. Ein Ausgangsbegriff ist der einer Klassifikation. Diese wird definiert wie folgt: ${ }^{15}$

$$
\begin{aligned}
& \text { Definition } A \text { classification } A \text { is a structure }<\operatorname{tok}(A) \text {, typ }(A),:_{A}>\text { consisting of } \\
& \text { a set of tokens tok }(A) \text {, a set of types typ }(A) \text { and a relation }:_{A} \text { on tok }(A) \times \\
& \text { typ }(A) \text {. For } t \in \operatorname{tok}(A) \text { and } \phi \in t y p(A) \text {, we say } t \text { is classified by } \phi \text { in } A \text {, if } t:_{A} \\
& \phi \text { holds. }
\end{aligned}
$$

Eine Klassifikation A kann durch die Auswahl der Elemente von tok(A) und typ(A) sehr spezifisch konstruiert werden. (Im Zusammenhang mit den obigen Wortfeldbeispielen würde einen nichts daran hindern, die Menge von Typen typ(A) nach Dimensionen zu strukturieren.)

Ein Channel stellt eine Verbindung zwischen zwei Klassifikationen A und B her; seine Aufgabe ist, den Informationsfluss zwischen A und B zu spezifizieren. Ein Channel wird formal selbst als eine Klassifikation definiert; seine Token sind sog. connections, ${ }^{16}$ Paare von einem Token aus A und einem Token aus B."

Definition Let $A$ and $B$ be classifications. A channel $C: A \Rightarrow B$ linking $A$ and $B$ is a classification $<$ tok $(C)$, typ $(C),:>$. The tokens of $C$ are connections, denoted $s \mapsto s^{\prime}$, with $s \in \operatorname{tok}(A)$ and $s^{\prime} \in \operatorname{tok}(B)$. The types of $C$ are constraints, denoted $\phi \rightarrow \psi$, with $\phi \in \operatorname{typ}(A)$ and $\psi \in \operatorname{typ}(B)$.

Wird in einem Channel $\mathrm{C}$ ein Token $\mathrm{s} \mapsto \mathrm{s}^{\prime}$ durch einen constraint $\phi \rightarrow \psi$ klassifiziert (notiert wie in (48)),

$$
s \mapsto s^{\prime}: \phi \rightarrow \psi
$$

heisst das, dass die Tatsache, dass s : $\phi$ die Information trägt, dass s' : $\psi$. Nützlich ist auch eine 2-dimensionale Darstellung dieser Aussage, in der die beiden Ebenen von Tokens und Types deutlich werden, vgl.

$$
\begin{gathered}
\phi \rightarrow \psi \\
: \\
\mathrm{s} \mapsto \mathrm{s}^{\prime}
\end{gathered}
$$

Cavedon/Glasbey (1994) nutzen Channels, um generische Sätze zu interpretieren. Ohne auf ihre Argumentation genauer einzugehen, seien für ihre Wahl von Channels als Schemata zur Deutung solcher Sätze zwei Punkte angeführt:

15 Ich entnehme diese Definition und die in (47) dem Papier von Cavedon/Glasbey (1994), vgl. dort p. 9.

16 Cavedon/Glasbey (1994) machen auf eine Vereinfachung aufmerksam, die sie mit dieser Charakterisierung der Token eines channels vornehmen, vgl. ihre Fn. 11.

17 Spezifische Connections sind aus der Situationssemantik bekannt; die Verwendung eines Eigennamens in einer Äußerungssituation (ein Eigennamen-Token) und sein Referent, ein Objekt in einer beschriebenen Situation, wurden als durch eine connection verbunden dargestellt. Damit sollte ein Zugriff auf Eigennamen-Referenten modelliert werden, der ohne Benutzung von dessen möglichen Eigenschaften auskam. Vgl. Barwise/Perry (1983), Gawron/Peters (1990). 
- Generische Aussagen könnte implizite kontextuelle Einschränkungen enthalten.

- Generische Aussagen können als Artikulation eines constraints auf der Typen-Ebene gedeutet werden, sind dagegen implizit in Bezug auf die Objekte (Token), für die der constraint gilt.

Der erste Punkt wird dadurch illustriert, dass (50.a) und (50.b) beide als gleichzeitig wahr angenommen werden können:

(50) (a) Peacocks lay eggs.

(b) Peacocks have brightly coloured tail-feathers.

Beide generischen Aussagen gelten für verschiedene Typen von Tieren, (a) nur für die Weibchen, (b) nur für die Männchen. Der zweite Punkte ist damit plausibel gemacht, dass generische Aussagen sich dadurch auszeichnen, dass sie Ausnahmen implizit lassen; (50.a) ist z.B. implizit in Bezug auf zu junge (weibliche) Exemplare.

Die Deutung einer Aussage wie (50.a) involviert nach Cavedon/Glasbey einen expliziteren Channel $C^{\prime}$, der den constraint PEACOCK\&FEMALE $\rightarrow$ LAY_EGGS enthält. Bei diesem Channel sind die connections von der Form $t \mapsto t$, d.h. sie bilden ein Token $t$ auf es selbst ab. Die Nichtberücksichtigung von Ausnahmen kann durch die Annahme eines 'normality constraints' für den Channel beschrieben werden.

Channels als Interpretionsschemata sind auch für die Deutung von E-Satz-Sequenzen interessant. Das Auftreten von Satztopiks stellt dabei eine Komplexität dar, die mit Elaboration interagiert, aber wie oben festgestellt, für die Diskursrelation selbst nicht notwendig ist. Im Zusammenhang mit den Arbeiten von Kim waren oben bereits Situationen und Individuen als Tokens von Klassifikationen dargestellt worden. Für die anvisierte Channel-Konstruktion geht es um die Rolle, die ein solches Token gleichzeitig in der ESatz-Deutung wie in der K-Satz-Deutung spielt. Die Idee ist, dass es das Objekt ausmacht, das K- und E-Deutung miteinander teilen.

Der Text Wetter_2 liefert hierzu ein einfaches Beispiel:

Wetter_2:

(43) (K) Es regnete.

(E1) Es fielen ungewöhnlich dicke Tropfen.

In (K) und (E1) kommen keine Satztopiks vor, vielmehr drücken beide Austin'sche Propositionen aus, d.h. klassifizieren eine Situation. Es ist plausibel, dass es in beiden Propositionen die gleiche Situation ist; sie sei - als durch den E-Satz klassifizierte Situation mit $\mathrm{s}_{\mathrm{e}}$ bezeichnet. Eine mit Elaboration einhergehende Intuition ist nun, dass $(\mathrm{K})$ von (E1) impliziert wird, auch so ausdrückbar: soll sich (E1) auf (K) per Elaboration beziehen, setzt es die Wahrheit von $(\mathrm{K})$ voraus. Dies lässt sich durch einen constraint darstellen, der die deskriptive Information dieses Zusammenhanges enthält:

\section{FALLEN UNGEWÖHNLICH DICKE TROPFEN $\rightarrow$ REGNET}

Dass ein Zusammenhang zwischen (E1) und (K) in (43) per Elaboration herstellbar ist, kann nun durch Annahme eines Channels erklärt werden. In einem ersten Ansatz ließe sich sagen, dass es das Token $s_{e} \mapsto s_{e}$ ist, das durch den constraint in (51) klassifiziert wird: 


\section{$\left(s_{\mathrm{e}} \mapsto \mathrm{s}_{\mathrm{e}}\right)$ : (FALLEN UNGEWÖHNLICH DICKE TROPFEN $\rightarrow$ REGNET)}

Diese Darstellung ist allerdings in zwei Hinsichten zu entwickeln. Eine ist, dass im angenommenen constraint nicht herauskommt, welcher spezifische Aspekt der K-Deutung durch die (E1) angesprochen wird. Eine solche Spezifik wollen wir aber isolieren, da auf ihr - bei Hinzukommen weiterer E-Sätze - die Dimension der Elaboration zu definieren ist. Unten wird diese Spezifik dadurch berücksichtigt, dass ein solcher constraint unter Benutzung einer K-Deutung formuliert wird, die in einem einschlägigen Aspekt modifiziert ist. Ein solcher constraint hat dann ein Schema wie in (53), wobei e ein aus der ESatz-Deutung abgeleiteter Typ ist und $\mathrm{k}^{\prime}$ ein Typ, der die Modifizierung der K-SatzDeutung berücksichtigt.

$$
\left(\mathrm{s}_{\mathrm{e}} \mapsto \mathrm{s}_{\mathrm{e}}\right):\left(\varepsilon \rightarrow \mathrm{\kappa}^{\prime}\right)
$$

Der in (52) benutzte constraint ist ausserdem in einen Zusammenhang zu stellen, der seine Leistung für die Inferenz der Diskursrelation Elaboration explizit macht. Im unserem Beispiel, Wetter_2, gibt es nur einen einzigen E-Satz, aus dem das Vorliegen von Elaboration zu abzuleiten ist. Wir können diese Inferenzmöglichkeit durch ein constraintSchema charakterisieren, das zwei Dinge in Verbindung bringt: die Klassifikation von $\mathrm{s}_{\mathrm{e}}$ wie in (52) und die Tatsache, dass die K-Satz-Deutung elaboriert wird. Letztere Tatsache drücken wir dadurch aus, dass die durch $(\mathrm{K})$ klassifizierte Situation $\mathrm{s}_{\mathrm{k}}$ vom Typ ELABORATED ist, aus dem die eigentliche Relation Elaboration abzuleiten ist. Wir haben es dann mit einem Token $s_{\mathrm{e}} \mapsto \mathrm{s}_{\mathrm{k}}$ zu tun. Sei $\varepsilon$ ein Typ, der aus der E-SatzDeutung abzuleiten ist (vgl. etwa oben den Antezedent in (51)), und $\kappa^{\prime}$ ein Typ, der aus einer K-Satz-Deutung abzuleiten ist, die hinsichtlich einer Dimension modifiziert ist (vgl. Abschnitt 7.3.2). Dann gilt für die Verarbeitung von (E1) folgender Channel für Elaboration:

$$
\left(s_{\mathrm{e}} \mapsto s_{\mathrm{k}}\right):\left(\varepsilon \& \kappa^{\prime} \rightarrow \text { ELABORATED }\right)
$$

D.h. $\mathrm{s}_{\mathrm{e}}$ muss, das wird in (53) illustriert, sowohl vom Typ $\varepsilon$ wie vom Typ $\mathrm{k}$ sein; $\mathrm{s}_{\mathrm{k}}$ ist dagegen vom Typ ELABORATED. Beim zweiten hinzukommenden E-Satz muss die in $\kappa '$ angesprochene Dimension weiter spezifiziert werden, vgl. unten Abschnitt 7.3.4.

Vor der weiteren Entwicklung dieser Modellierungsidee sei darauf hingewiesen, dass mit der Konstruktion von Channels eine Maxime von SDRT nicht beachtet wird, nämlich die, die Repräsentation satzinterner Information (per DRSen) von der Repräsentation ihrer pragmatischen Rolle - gerade für die Konstruktion von Diskursrelationen - in ihrem Darstellungsformat zu unterscheiden. Eine Entsprechung zu den constraints von Channels besteht in SDRT in den Axiomen des DICE-Moduls, in welche Information aus den DRSen durch Übersetzung eingeht. Konstruktion von Channels sehe ich aber im vorliegenden Zusammenhang als nützlich an, da so die Beziehung zwischen Satz- und Diskurstopik gleichzeitig sichtbar gemacht werden kann. Die Frage nach der Beziehung 
zwischen constraints und DICE-Axiomen ist zudem für sich interessant, wenn sie auch hier nicht weiter verfolgt werden soll. ${ }^{18}$

\subsection{Typen}

Noch in anderer Weise haben wir mit dem Ausgangspunkt in SDRT zurecht zu kommen. Die Deutungen von Satzvorkommen in Texten werden dort als DRSen modelliert, denen Objekte mit dem Status von Propositionen entsprechen. Innerhalb von Channels haben dagegen die Objekte mit deskriptiver Deutung den Status von Typen, d.h. von Funktionen in Propositionen. Allerdings können wir mit Cooper (1993) DRSen eine situationstheoretische Deutung als mehrstellige Typen geben. Die Idee ist dort, dass DRSen als Objekte aufgefaßt werden können, die durch simultane Abstraktion über eine Menge von Parametern im Sinne von Aczel/Lunnon (1991) definiert sind. In einem $\lambda$-Präfix wird, zwecks semantischer Differenzierung, dann nicht nur eine Menge von Parametern spezifiziert, die den Diskursreferenten einer DRS entspricht, sondern eine Abbildung von Rollenindizes auf Parameter (Cooper (1993)). Eine solche Abbildung wird assignment genannt. Ein n-stelliger Typ wird entsprechend durch ein n-stelliges assignment abgesättigt (vgl. hierzu Cooper (1991)).

Die situationstheoretische Repräsentation solcher Typen wird im folgenden Abschnitt dargestellt. Ziel ist es, in einem constraint des Aufbaus $\phi \rightarrow \psi$ die Konstituenten $\phi$ und $\psi$ spezifizieren zu können, die auch ihrerseits den Status von Typen haben. Zunächst soll das nötige situationstheoretische Vokabular vorgestellt werden.

\subsubsection{Repräsentation von situationstheoretischen Objekten}

Für die in Abschnitt 5 verwendete 'lineare' Repräsentation, das s vom Typ $\tau$ ist, vgl. (55), verwenden wir im folgenden eine 2-dimensionale Notation (Extended Kamp Notation, $E K N$, vgl. Barwise/Cooper (1993)) in (56): ein Token s wird in einem Feld links oben in einem Kasten notiert, der den 'internen' Typ $\tau$ enthält:

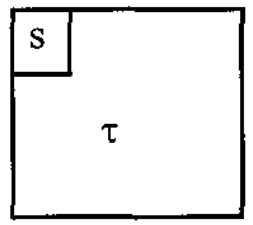

Ein konkretes Beispiel für diese Notation ist (25.a) aus Abschnitt 5. Das Token ist dort 'oracle ${ }_{x}$ ', der interne Typ erhält in EKN einen expliziten Rollenindex, hier einfach 'argument ${ }^{\prime}$.

oracle $_{\mathrm{X}}: \mathrm{RUND}(\mathrm{x})$

18 Auf einen semantischen Effekt, der durch den Unterschied an Granularität zwischen Channels und Ashers nichtmonotoner Implikation entsteht, weist Glasbey 1996 (dort Abschnitt 4) hin. 
(57)

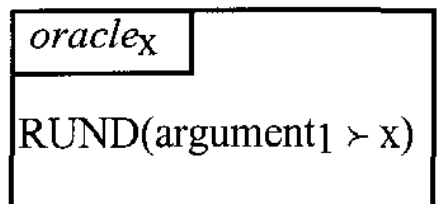

Rollenindizes werden auch bei Abstraktion eingeführt, vgl. die Darstellung des Typs (25.b) in (58), in dem der Parameter x der Wert für einen Rollenindex object ist:

(25.b) $\lambda \mathrm{x}\left[\right.$ oracle $\left._{\mathrm{X}}: \mathrm{RUND}(\mathrm{x})\right]$

$$
\begin{array}{|l|}
\hline \multicolumn{2}{|l|}{\text { object } \succ \mathrm{x}^{\text {oracle }_{\mathrm{X}}}} \\
\hline \mathrm{RUND}\left(\operatorname{argument}_{1} \succ \mathrm{x}\right) \\
\hline
\end{array}
$$

Anwendung von Typen auf Argumente geschieht unter Identifikation der Rollenindizes. Dass ein Token von einem bestimmten Typ ist, wie angegeben in (25.c), impliziert, dass es der Wert für die gleiche Rolle eines Typs und eines assignments ist, vgl. (59).

$$
\mathrm{m}: \lambda \mathrm{x}\left[\operatorname{oracle}_{\mathrm{X}}: \mathrm{RUND}(\mathrm{x})\right]
$$

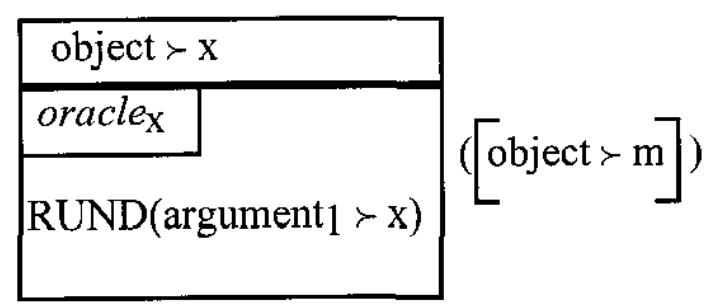

Anwendung eines Typs auf ein Argument führt bei linearer Repräsentation zur Tilgung des $\lambda$-Präfixes und Einsetzung des Arguments; in EKN enspricht der Tilgung des Präfixes die Tilgung der assignment-Leiste, vgl. (60), (61):

$$
\text { oracle }_{\mathrm{m}}: \mathrm{RUND}(\mathrm{m})
$$

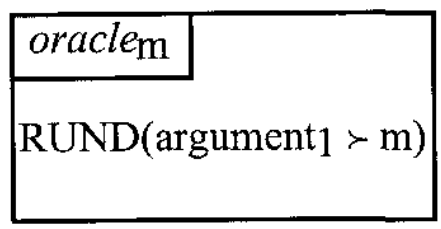

In EKN können semantische Beschränkungen auf Propositionen durch Information in einer Restriction Box dargestellt werden. In (62) sind $\mathrm{p}$ und $\mathrm{q}$ Propositionen; $\mathrm{p}$ ist nur dann wahr oder falsch, wenn (die Proposition in der Restriction Box) $q$ wahr ist: 
$(62)$

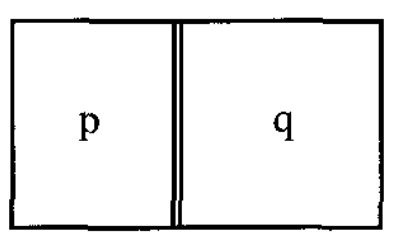

\subsubsection{Modifikation der K-Satz-Deutung}

Der in (51) angegebene constraint

$$
\text { FALLEN UNGEWÖHNLICH DICKE TROPFEN } \rightarrow \text { REGNET }
$$

lässt sich mit den Repräsentationsmitteln aus dem vorigen Abschnitt darstellen wie in

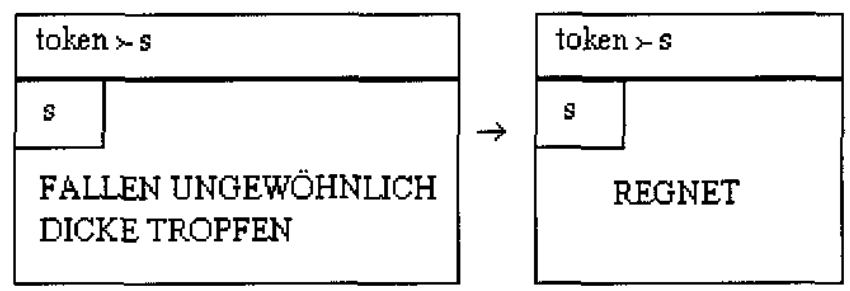

Unbefriedigend an diesem constraint ist, dass er auf Dimensionsinformation keinen Bezug nimmt. Das Fallen ungewöhnlich großer Tropfen spezifiziert sicher einen Aspekt des Sukzedent-Typs in (63).

Dimensionen sind sicher ein Hintergrund-Phänomen, insofern als Bestandteile einer Restriction Box darstellbar. Der hier vorgenommene Schritt ist, durch eine Dimension den Sukzedent-Typ in geeigneter Weise einzuschränken. Intuitiv liefert (E1) im vorhandenen Text nicht mehr, als dass es auf eine bestimmte Weise regnet. Statt dem Sukzedenten in (63) ist daher ein Typ wie in (64) anzusetzen, in dem s von einem um eine Rolle erweiterten (internen) Typ REGNET(manner $\succ m$ ) klassifiziert wird, der seinerseits Bestandteil einer Dimension $\delta$ ist.

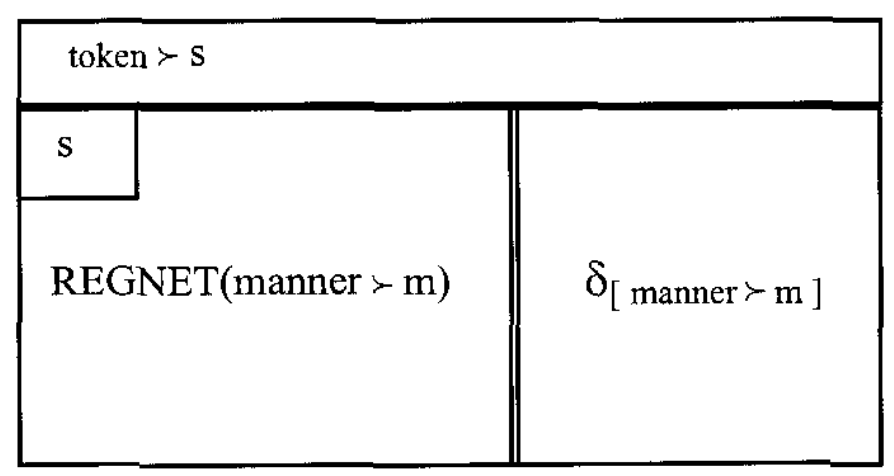

Die zusätzliche Rolle für den internen Typ (hier REGNET) ist identisch mit einer Position, die die Dimension d vergibt. Die Beziehung zwischen dem aus $(\mathrm{K})$ allein ableitbaren internen Typ ('REGNET') und dem um eine Rolle erweiterten Typ ('REGNET(manner m)') kann semantisch in Anlehnung an eine Idee von J. Barwise als Explizitmachung einer Dimension of Change angesehen werden, die in der durch die Äußerung von (K) al- 
lein klassifizierten Situation $\left(\mathrm{s}_{\mathrm{k}}\right.$ ) latent war und aufgrund dieser Äußerung nicht diskriminiert wurde. ${ }^{19}$ Erst die Artikulation von (E1) fügt dieser Situation eine Klassifizierung auch nach dieser Dimension hinzu und verfeinert ('elaboriert') sie in diesem Sinn.

Eine verallgemeinerte Darstellung des Sukzedenten gibt dann (65). Dort ist $\tau_{\kappa}$ der aus der K-Satz-Deutung hervorgehende interne Typ ('REGNET' im obigen Beispiel); ' $\tau_{\mathrm{K}}+\left[\right.$ position $\left._{\mathrm{n}} \succ \mathrm{x}_{\mathrm{n}}\right]$ ' ist der um das Argument mit dem Rollenindex 'position $\mathrm{n}_{\mathrm{n}}$ ' erweiterte interne Typ. Dieser wird durch Hintergrundinformation festgelegt, d.h. durch die Dimen$\operatorname{sion} \delta$.

\begin{tabular}{|c|c|}
\hline token $\succ \mathrm{s}$ & \\
\hline $\mathrm{s}$ & \\
\hline$\tau_{K}+\left[\operatorname{position}_{n} \succ x_{n}\right]$ & $\delta\left[\operatorname{position}_{\mathrm{n}} \succ \mathrm{x}_{\mathrm{n}}\right]$ \\
\hline
\end{tabular}

\subsubsection{Dimensionen}

Auch die Dimension $\delta$ wird als ein Typ konstruiert. Dabei ist wichtig, durch die Wahl geeigneter Rollenindizes im assignment die Aspekte der relevanten Klassifikationen auszuzeichen. Eine Dimension ist auch dadurch inhaltlich festgelegt, dass sie über ein gegebenes Objekt definiert ist, in unseren Fällen ist dies die Situation oder das Individuum, das durch die E-Sätze elaboriert wird. Dieses Objekt o hat die Rolle eines 'Rahmens' und erhält den Rollenindex frame.

Insgesamt leistet eine Dimension eine Klassifikation einer Sequenz von Objekten $x_{1}$, $x_{2}$, etc. anhand einer Sequenz von kontrastierenden Eigenschaften PROPERTY, $P R O P E R T Y_{2}$ etc. Die Objekte sind zugänglich über Rollenindizes position 1 , position 2 , etc.

Ohne Rahmen, Objekte und Eigenschaften weiter semantisch einzuschränken ${ }^{20}$ ergibt sich im allgemeinen für eine Dimension $\delta$ ein Objekt wie in

19 Ich verwende hier eine Idee von Jon Barwise (1989) zur Interpretation des Kontrastes von It's 4 o'clock vs. It's 4 o'clock here. Latente Rollen sind nach seiner Darstellung mit dem Vorhandensein einer Perspektive verbunden. Stimmt meine Analyse, dann enthüllt (E1) eine Perspektive, die in (K) allein latent ist.

20 Durch Einschränkungen auf die Eigenschaften kann (und muss eigentlich) Kontrastivität verlangt werden. 
(66)

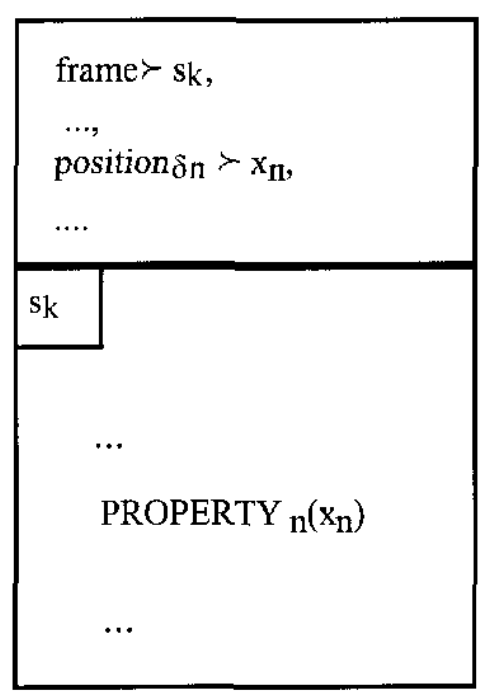

$\{\delta\}$

Als Typ ist ein solches Objekt weder wahr noch falsch, kann deswegen nicht als einschränkende Proposition fungieren wie q in (62) oben. Aus $\delta$ lässt sich jedoch eine Proposition definieren, indem es auf ein assignment angewendet wird, vgl.

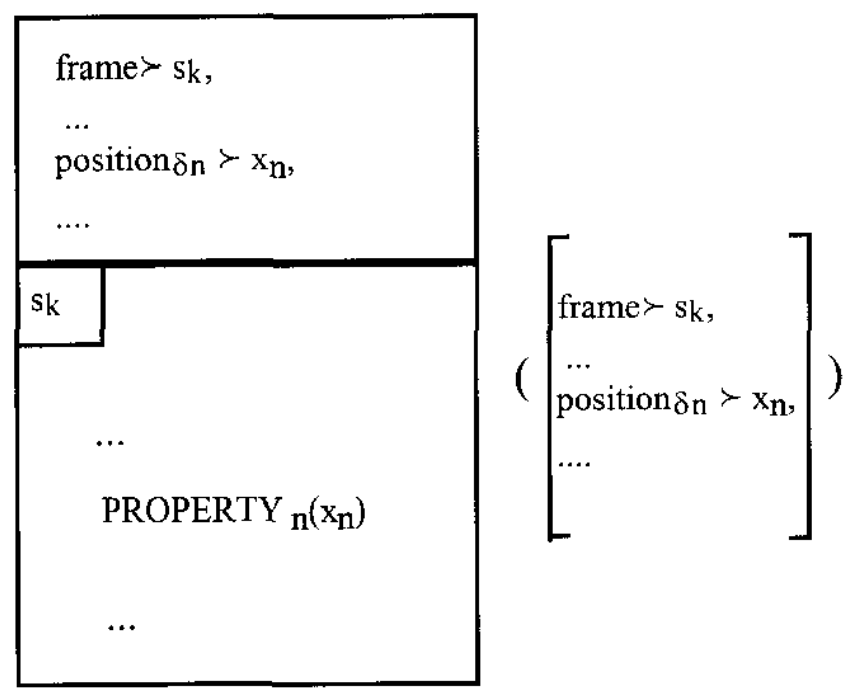

$\{\delta\}$

Ein Objekt dieser Struktur hat Propositions-Status und kann in der Restriction Box eines constraint-Sukzedenten stehen. Ein inkrementell orientiertes Vorgehen ist nun, im Fall des ersten E-Satzes auch $\delta$ nur mit einer Position auszustatten. Es leistet nicht mehr als die oben dargestellte Einführung einer bisher latenten Dimension.

Wir setzen dann für die Verarbeitung des ersten E-Satzes einen constraint an wie in

(68) $\varepsilon \rightarrow \kappa_{+} 1 \delta$ (klassifiziert wird die connection $\mathrm{s}_{\mathrm{e}} \mapsto \mathrm{s}_{\mathrm{e}}$ ) 
Dabei ist $\varepsilon$ der Typ, den die E--Satz-Deutung ergibt; $\kappa_{+} \mathrm{rn} \delta$ ist ein Typ, der aus der KSatz-Deutung durch Erweiterung um eine 'interne Rolle' hervorgeht, vgl. oben (65). Darin ist $\kappa_{+} 1 \delta$ ein Objekt von folgender Struktur:

(69)

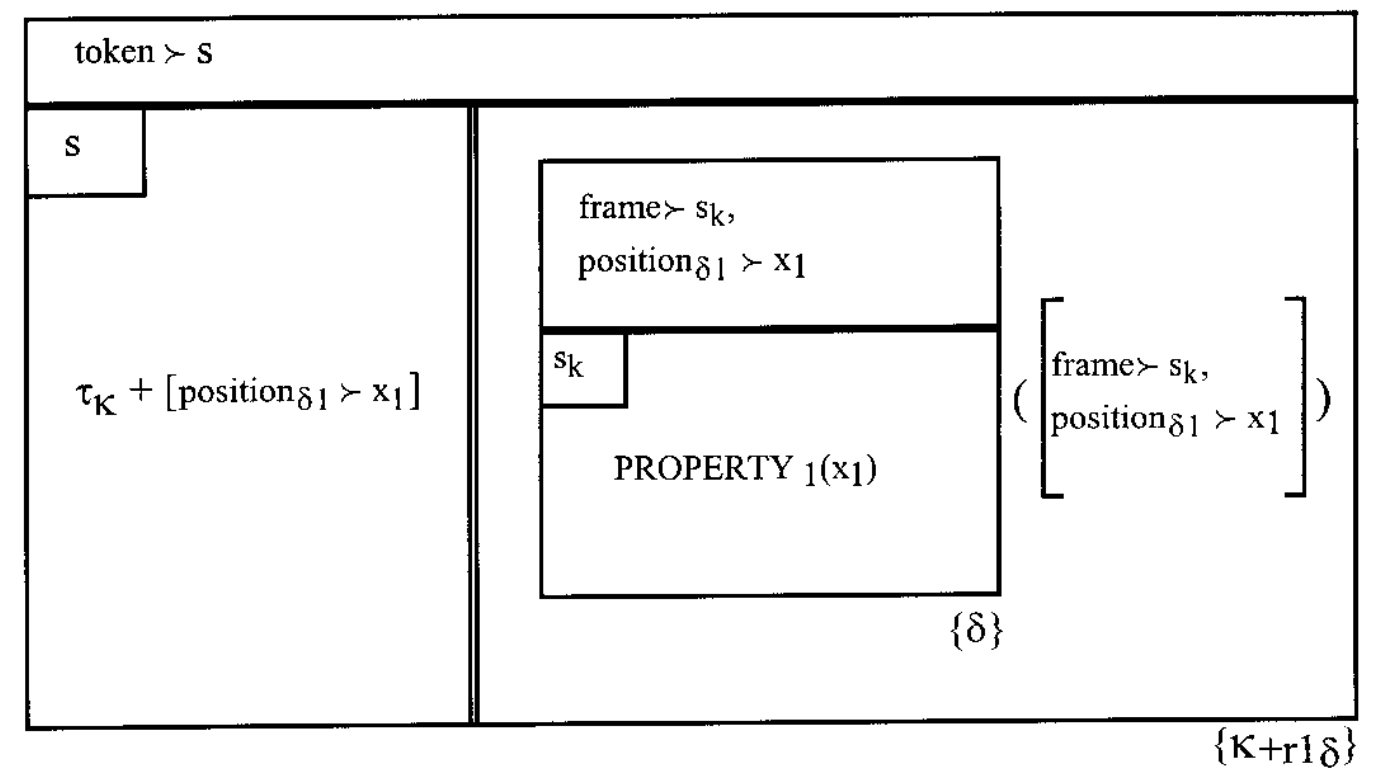

\subsubsection{Constraints für den zweiten E-Satz}

Ein Beispiel für einen Text mit einem zweiten E-Satz, in dem keine Satztopiks auftreten, ist

Wetter 1:

(38) $(\mathrm{K})^{-}$Es war gutes Wetter.

(E1) Es war nicht zu kalt.

(E2) Und es war nicht zu trocken.

Ein constraint für die Wahl der K-Deutung als attachment site bei der Verarbeitung von (E2) ist

(70) $\varepsilon \rightarrow \kappa_{+} 2 \delta \quad$ (klassifiziert wird die connection $\mathrm{s}_{\mathrm{e}} \mapsto \mathrm{s}_{\mathrm{e}}$ )

Dabei ist $\kappa_{+} 2 \delta$ ist wie in (71); dort ist die Dimension $\delta^{\prime}$ bereits in Bezug auf eine Position durch einen Wert $\mathrm{b}$ festgelegt (das Ergebnis der Verarbeitung des ersten E-Satzes). 
(71)

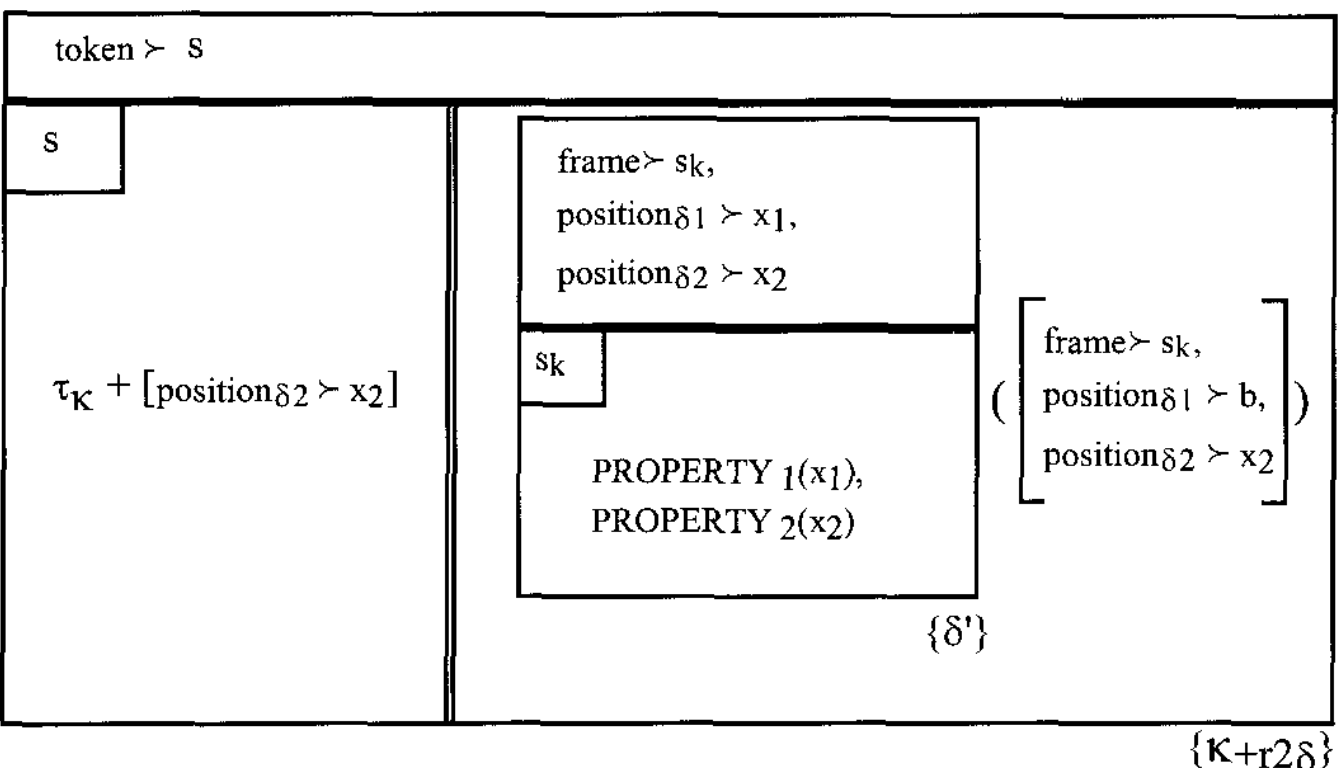

\subsubsection{Constraints für Satztopiks}

Ein Beispiel für einen Text mit einem Satztopik im ersten E-Satz ist

Schulwahl_I_A:

(29) (K) Nach dem 6. Schuljahr suchen die Freunde von Meryem verschiedene Schulen auf.

(E1) So wird [Hamdi] $\mathrm{T}$ glücklicherweise aufs Gymnasium gehen.

Ein constraint für die Wahl von (K) als attachment site ist (72); entsprechend zu den Überlegungen in Abschnitt 6 oben wird angenommen, dass die Satztopik-Referenten Token für den constraint liefern.

(72) $\varepsilon \rightarrow \kappa_{+\mathrm{r} 1 \delta} \quad$ (klassifiziert werden die connections $\mathrm{s}_{\mathrm{e}} \mapsto \mathrm{s}_{\mathrm{e}}$ und $\left.\mathrm{a} \mapsto \mathrm{a}\right)$

Die an diesem constraint beteiligten Typen klassifizieren beide eine Situation $\mathrm{s}$ und ein Objekt o, den Referenten des Satztopiks. Die Idee, dass die Satztopik-Referenten die 'Indizes' hergeben, anhand von denen die Elaborations-Dimension konstruiert ist, wird so berücksichtigt, dass im Sukzedenten des constraints der Wert für das Objekt o an die Dimension über das assignment vererbt wird. Der constraint hat eine Struktur wie in (73): 
(73)

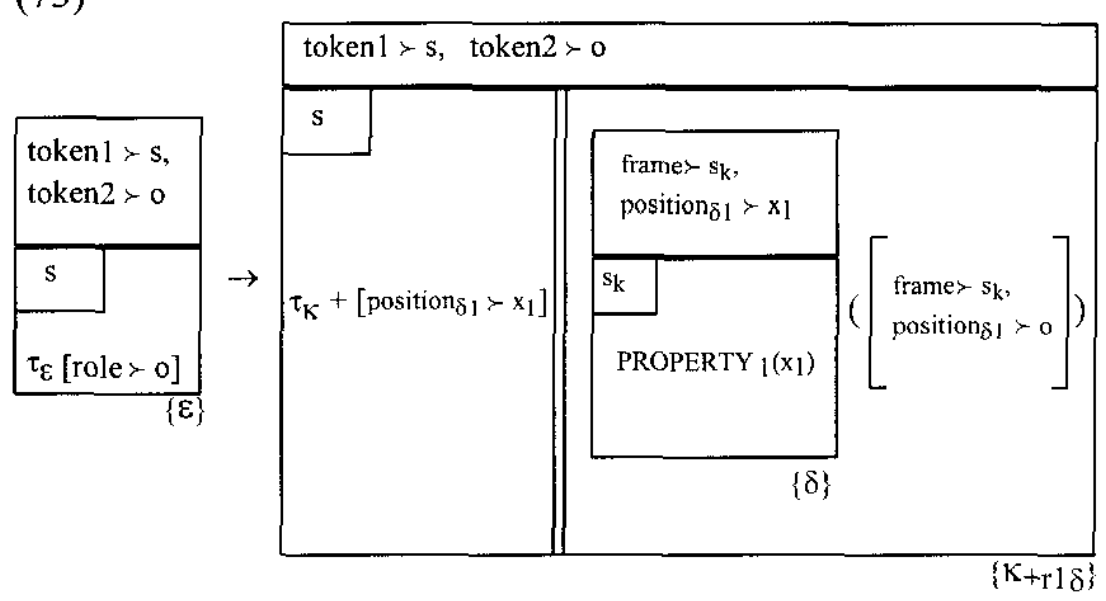

Durch Anwendung auf die Token $\mathrm{s}_{\mathrm{e}} \mapsto \mathrm{s}_{\mathrm{e}}$ und $\mathrm{a} \mapsto$ a erhält man eine Relation zwischen zwei aus (E1) und (K) abgeleiteten Propositionen:

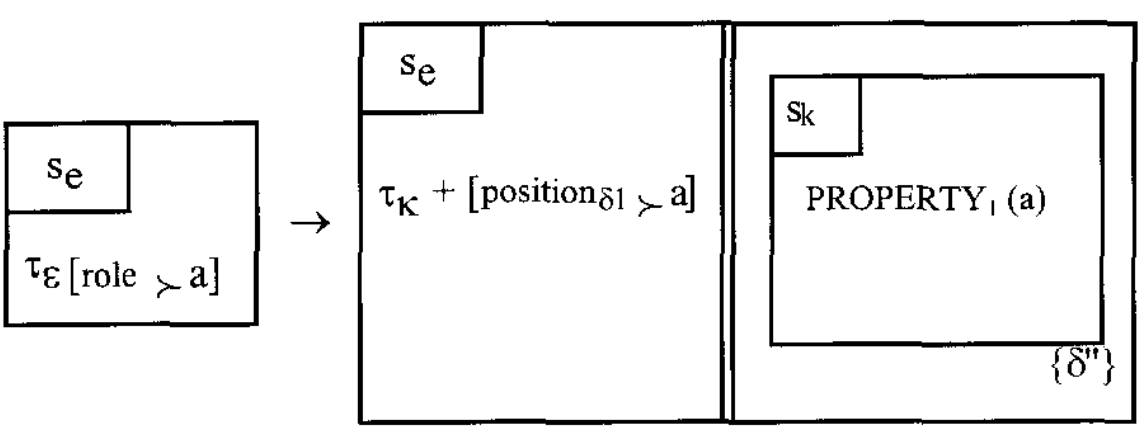

Die relevante in dieser Konstruktion enthaltene These ist, dass der Satztopik-Referent a eine durch die Dimension $\delta^{\prime \prime}$ ausgezeichnete Eigenschaft innehat, die eine Verfeinerung der Deutung von K leistet. Satztopiks kommt somit in Elaboration-Kontexten die pragmatische Funktion zu, direkt den Wert der Rollenindizes der Dimension festzulegen, nach der die Deutung von $(\mathrm{K})$ verfeinert wird.

\section{Schluss}

Als Abschluss sei ein Rückblick auf die updating-Prinzipien von SDRT, dem nach wie vor intendierten Rahmen der gemachten Überlegungen, erlaubt. Diese lauteten:

1. Der Inhalt eines neu verarbeiteten Satzes erhält die Rolle einer Diskurskonstituente, die, anders als in der klassischen DRT, 'segmentiert' bleibt, d.h. nicht mit dem Inhalt vorhergehender Sätze verschmolzen wird.

2. Bei der Anknüpfung einer jeden neuen Diskurskonstituente $\beta$ wird die Spezifizierung einer Diskursrelation $\mathrm{R}$ gefordert.

3. Im vorhergehenden Text wird eine Diskurskonsituente $\alpha$ (attachment site) ausgewählt, an die $\beta$ durch $R$ angeknüpft wird und

4. $\beta$ wird zu Zwecken der Kohärenz mit $\alpha$ ggf. um zusätzliche Information $\phi$ angereichert. Das heißt, durch Ausnutzung von Weltwissen wird eine angereicherte Konstituente $\beta$ ' inferiert. 
Davon wurde Prinzip 1. nicht weiter diskutiert, sondern dadurch erfüllt, dass die E- und K-Satz-Deutungen durchweg als isolierte Objekte (Propositionen oder Typen) aufgefasst wurden. Prinzip 2. wurde dadurch angesprochen, dass es im empirischen Kapitel 6. und im Modellierungskapitel 7. stets um das Vorliegen einer Diskursrelation ging, Elaboration. Im Papier wurde allerdings kaum die Wahl einer alternativen Diskursrelation diskutiert.

Prinzip 3. wurde dadurch angesprochen, dass das Vorliegen von bestimmten constraints (siehe die Diskussion von (68), (70 und (72)) als Voraussetzung für die Wahl einer Diskurskonstituente $\|\mathrm{K}\|$ von einem aktualen $(\mathrm{E})$ aus angesehen wurde. Hier Empirische Arbeit wurde hier nur in kleinstem Umfang geleistet, eher ging es um eine Darstellung der schematischen Bedingungen.

Das Prinzip 4. wurde schließlich in der Weise inhaltlich ausgefüllt, als die Konstruktionen der einzelnen Channels gerade Information darstellen, mit denen ein aus (E) abgeleiteter Antezedent eines constraints ausgestattet werden muss, damit die Relation Elaboration inferiert werden kann.

\section{Literaturverzeichnis}

Aczel, P.; R. Lunnon. (1991): Universes and Parameters. In: J. Barwise et al. (eds.) Situation Theory and its Applications. Vol 2. CSLI Lecture Notes.

Asher, N. (1993): Reference to Abstract Objects in Discourse. Kluwer.

- (1995): From Discourse Macro-structure to Micro-structure and Back again: Discourse Semantics and the Focus/Background Distinction. Proceedings of the Workshops in Prague, February 1995. Bad Teinach, May 1995. Vol. I, 21-51.

- $\quad$; A. Lascarides. (1998): Bridging, Journal of Semantics, 15.1, 83-113.

Barwise, J.;R.Cooper. (1993): Extended Kamp Notation: A Graphical Notation for Situation Theory. In: P.Aczel et al. (Hg.) Situation Theory and its Applications. Vol.3. CSLI Lecture Notes 37. Stanford. 29-54.

Barwise, J.; J. Perry. (1983): Situations and Attitudes. Bradford Books. MIT Press. Cambridge/Mass.

Büring, D. (1998): Focus and Topic in a Complex Model of Discourse. Ms. Universität Köln.

Cavedon, L.; S. Glasbey. (1994): Outline of an Information-Flow Model of Generics. Ms. Ersch. in Acta Linguistica Hungarica.

Cooper, R. (1991): A working person's guide to situation theory. To appear in: S. L. Hansen; F. Sörensen (eds.) Semantic Representation and Interpretation. Frederiksberg. Samfundslitteratur. 1992.

(1993): Towards a general semantic framework. Centre for Cognitive Science/ Human Communication Research Centre. Universität Edinburg. Deliverable zu ESPRIT BR Project 6852, DYANA-2.

Devlin, K. (1991): Logic and Information. Cambridge University Press.

Dölling, J. (1994): Semantic Sorts and Systematic Ambiguity. In: Robering (ed.) (1994), 57-83.

v. Fintel, K. (1994): Restrictions on Quantifier Domains. Ph. Diss. Univerv. of Amherst.

Frey, W. (2000): Über die syntaktische Position des Satztopiks im Deutschen. In diesem Band.

Gawron, J.M.; S. Peters. (1990): Anaphora and Quantification in Situation Semantics. CSLI Lecture Notes 19. Stanford.

Glasbey, S. (1994): Event Structure in Natural Language Discourse. PhD. Thesis. Universität Edinburg.

Glasbey, S. (1996): The Progressive: A Channel Theoretic Analysis. Erscheint in Journal of Semantics.

Grosz, B.; C. Sidner. (1986): Attention, Intentions, and the Structure of Discourse. ACL 12, 175-204.

Kamp, H.; U.Reyle. (1993): From Discourse to Logic. Kluver. Dordrecht.

v. Kuppevelt (1995): Discourse structure, topicality and questioning. JL 31, 109-145.

Hobbs, J. (1985): On the Coherence and Structure of Discourse. Report No. CSLI-85-37, CSLI Stanford University.

; M.H. Agar. (1985): The Coherence of Incoherent Discourse. CSLI Report No. CSLI-85-38. Stanford.

Hockett, Ch. F. (1958): A Course in Modern Linguistics. New York. McMillan

Jacobs, J. (1999): The Dimensions of Topic-Comment. Papier Universität Wuppertal. 
Kamp, H. (1981): A Theory of Truth and Semantic Representation. In: G. Groenendijk; Th. Janssen; M. Stokhof. (eds.) Formal Methods in the Study of Language. Mathematisch Centrum, Amsterdam.

Kim, Y. (1998): Information Articulation and Truth Conditions of Existential Sentences. Language and Information, vol-1.1.

- (erscheint): A Situation Semantic Account of Topic vs. Nominative Marking. Proceedings of the ITALLC 98.

Krifka, M. (1992): A compositional semantics for muliple focus constructions. In: J.Jacobs (Hg.) Informationsstruktur und Grammatik. Linguistische Berichte, Sonderheft 4.

Lang, E. (1979): Zum Status der Satzadverbiale. Slovo a slovenost. Prag. Vol 3. 200-213.

Li, Ch. (ed.) (1976): Subject and Topic. Academic Press. New York.

Lutzeier, P. (1981): Wort und Feld. Niemeyer. Tübingen.

Mann, W.C.; Thompson, S.A. (1986): Rhetorical Structure Theory. In: Kempen, G. (ed.) Natural Language Generation. Martinus Nijhoff Publishers, 85-95.

Polanyi, L. (1985): A Theory of Discourse Structure and Discourse Coherence. Papers from the General Session at the Twenty-First Regional Meeting of the Chicago Linguistics Society, 25-27.

Portner, P.; K. Yabushita. (1998): The Semantics and Pragmatics of Topic Phrases. Linguistics and Philosophy 21. 117-57.

Reinhart, T. (1981): Pragmatics and linguistics: an analysis of sentence topics. Philosophica 27. 53-94.

Reinhart, T. (1995): Interface Strategies. OTS Working Papers, Utrecht University.

Robering, K. (ed.) (1994): Sorten, Typen und Typenfreiheit. Arbeitspapiere zur Linguistik. TU Berlin. Institut für Linguistik.

Schachter, P. (1976): The Subject in Philippine Languages: Topic, Actor-Topic, or None of the above. In: Ch. Li (ed.), 491-518

Vallduví, E. (1993): Information packaging: A survey. HCRC research report. Human Communication Research Centre. University of Edinburgh. Netzadresse: www.hcrc.ed.ac.uk/publications/.

Vallduví, E. (1994): The dynamics of information packaging. Research Report Centre for Cognitive Science. Universität Edinburgh. 